Supporting information for:

\title{
Quantitative measurement of cytosolic and nuclear penetration of oligonucleotide therapeutics
}

Kirsten Deprey ${ }^{1}$, Nefeli Batistatou ${ }^{1}$, Marjoke F. Debets ${ }^{2}$, Jack Godfrey ${ }^{3}$, Kirstin B. VanderWall ${ }^{2}$, Rebecca R. Miles ${ }^{2}$, Livia Shehaj ${ }^{1}$, Jiaxing Guo ${ }^{1}$, Amy Andreucci ${ }^{3}$, Pachamuthu Kandasamy ${ }^{3}$, Genliang Lư ${ }^{3}$, Mamoru Shimizu³, Chandra Vargeese ${ }^{3}$, and Joshua A. Kritzer ${ }^{1, *}$

${ }^{1}$ Department of Chemistry, Tufts University, Medford, Massachusetts 02155, United States

${ }^{2}$ Lilly Research Laboratories, Eli Lilly and Company, Indianapolis, Indiana 46285, United States

${ }^{3}$ Wave Life Sciences, Cambridge, Massachusetts 02138, United States

*corresponding author: joshua.kritzer@tufts.edu

\section{Table of Contents}

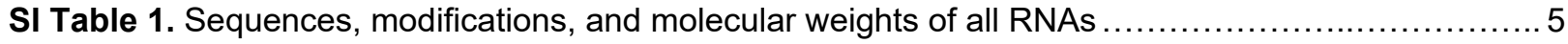

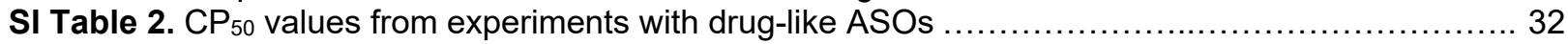

SI Table 3. $\mathrm{CP}_{50}$ values from CAPA experiments exploring the effects of patterning ..................... 32

SI Table 4. $\mathrm{CP}_{50}$ values from CAPA experiments with siRNAs .......................................... 32

SI Table 5. $\mathrm{CP}_{50}$ values from CAPA experiments in HeLa cells 24 hours after transduction ............... 33

SI Table 6. $\mathrm{CP}_{50}$ values from CAPA experiments in HeLa cells 48 hours after transduction ................33

SI Table 7. $\mathrm{CP}_{50}$ values from CAPA experiments in HeLa cells 72 hours after transduction ................33

SI Table 8. $\mathrm{CP}_{50}$ values from CAPA experiments in stable and transduced cell lines .................... 33

SI Table 9. $\mathrm{CP}_{50}$ values from time course CAPA experiments .......................................... 34

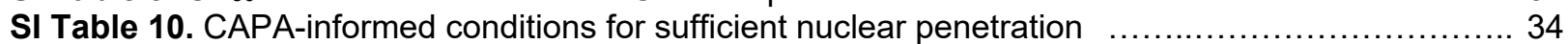

SI Table 11. $\mathrm{CP}_{50}$ values from CAPA experiments in HeLa and SH-SY5Y cell lines ..................... 34

SI Table 12. $\mathrm{CP}_{50}$ values for ASOs with different $5^{\prime}$ linkers in serum-free media ........................ 35

SI Table 13. $\mathrm{CP}_{50}$ values for ASOs with different $5^{\prime}$ linkers in full media ................................. 35

SI Table 14. $\mathrm{CP}_{50}$ values for ASOs with $5^{\prime}$ amide and click linkers in serum-free media ................. 35

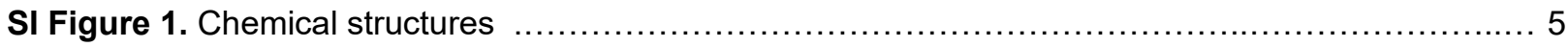

SI Figure 2. Application of CAPA to ASOs with sequences derived from the drug alicaforsen $\ldots \ldots \ldots \ldots$

SI Figure 3. Effects of sequence and length on cytosolic penetration $\ldots \ldots \ldots \ldots \ldots \ldots \ldots \ldots \ldots \ldots \ldots \ldots \ldots$

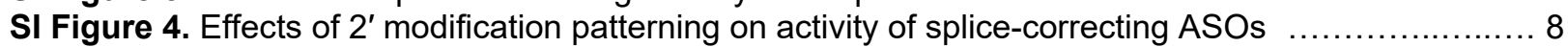

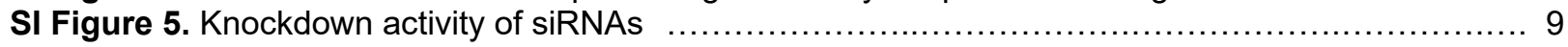

SI Figure 6. Knockdown activity of siRNAs after transfection using lipofectamine .................... 10

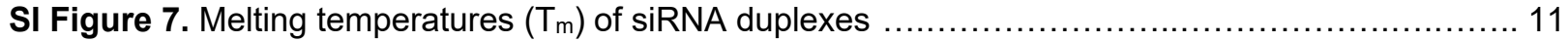

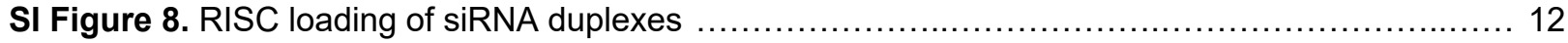

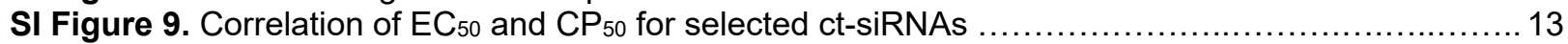

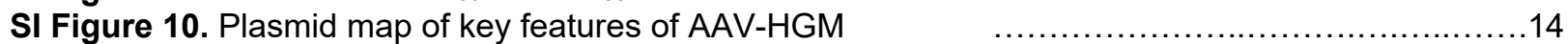

SI Figure 11. Sequence of the transgene open reading frame for the AAV-HGM plasmid ................15

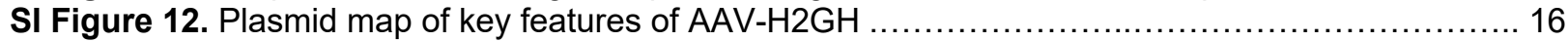

SI Figure 13. Sequence of the transgene open reading frame for the AAV-H2GH plasmid ............... 17

SI Figure 14. Transduction of HeLa cells with novel AAV vectors AAV-HGM and AAV-H2GH .......... 18

SI Figure 15. Benchmarking CAPA in AAV-transduced HeLa cells using control compounds ............. 19

SI Figure 16. Analyzing nuclear penetration and effects of serum for chemically modified RNAs ........ 20

SI Figure 17. Time course of nuclear localization of modified RNAs, grouped by incubation time ........ 22

SI Figure 18. Time course of nuclear localization of modified RNAs, grouped by compound ............. 23

SI Figure 19. Impact of chloroalkane linker chemistry on cytosolic localization ........................ 24

SI Figure 20. Splice-correction activity of selected ASOs after transfection using lipofectamine ..........26 
SI Figure 21. Splice-correcting activity of selected ASOs after gymnotic uptake

SI Figure 22. Transduction of SH-SY5Y cells with novel AAV vector AAV-H2GH ...................... 28

SI Figure 23. Chloroalkane-tagged ASOs are stable in serum-containing media ...................... 29

SI Figure 24. CAPA and knockdown activity in SH-SY5Y cells ................................... 30

SI Figure 25. Comparison of cytosolic localization for ASOs with amide and click linkers ............... 31

SI Table 1. Sequences, modifications, and molecular weights of all RNAs.

ct(PO): chloroalkane-phosphodiester-amide linker

ct(PS): chloroalkane-phosphorothioate-amide linker

ct(click): chloroalkane-phosphodiester-click linker

* : phosphorothioate backbone

m_ (bolded): 2'O-methoxyethyl group

$m$ _ (not bolded): 2'O-methyl group

C.: 5-methyl cytosine

\#: phosphoroamidite morpholino

f: 2'-fluoro

$S: S p$

$R: R p$

\begin{tabular}{|c|c|c|c|c|}
\hline & Sequence & $\begin{array}{c}\text { Extinction } \\
\text { coefficient } \\
\left(\mathrm{M}^{-1} \mathrm{~cm}^{-1}\right)\end{array}$ & $\begin{array}{c}\text { Expected } \\
\text { MW } \\
(\mathrm{g} / \mathrm{mol})\end{array}$ & $\begin{array}{l}\text { Observed } \\
\text { MW (g/mol) }\end{array}$ \\
\hline$A-1$ & $\operatorname{ct}(P O)-$ UCACUUUCAUAAUGCUGG & 159400 & 5851.4 & 5851.7 \\
\hline A-2 & $\operatorname{ct}(P O)-U^{*} C^{*} A^{*} C^{*} U^{*} U^{*} U^{*} C^{*} A^{*} U^{*} A^{*} A^{*} U^{*} G^{*} C^{*} U^{*} G^{*} G$ & 159400 & 6391.8 & 6393.4 \\
\hline A-3 & $\operatorname{ct}(P O)-m U m C m A m C m U m U m U m C m A m U m A m A m U m G m C m U m G m G$ & 175200 & 6124.4 & 6125.6 \\
\hline A-4 & $\begin{array}{l}\operatorname{ct}(P O)-m U^{*} m C^{*} m A^{*} m C^{*} m U^{*} m U^{*} m U^{*} m C^{*} \\
m A^{*} m U^{*} m A^{*} m A^{*} m U^{*} m G^{*} m C^{*} m U^{*} m G^{*} m G\end{array}$ & 175200 & 6664.9 & 6665.4 \\
\hline A-5 & $\operatorname{ct}(P O)-m T m \underline{C} m A m \underline{c} m T m T m T m \underline{C} m A m T m A m A m T m G m \underline{C} m T m G m G$ & 175200 & 7339.1 & 7340.8 \\
\hline A-6 & $\begin{array}{l}\operatorname{ct}(P O)-m T^{*} m \underline{C}^{*} m A^{*} m C^{*} m T^{*} m T^{*} m T^{*} m \underline{C}^{*} \\
m A^{*} m T^{*} m A^{*} m A^{*} m T^{*} m G^{*} m \underline{C}^{*} m T^{*} m G^{*} m G\end{array}$ & 175200 & 7611.7 & 7613.9 \\
\hline A-7 & $\begin{array}{l}\mathrm{ct}\left(\text { click) }-m T^{*} m \underline{C}^{*} m A^{*} m C^{*} m T^{*} m T^{*} m T^{*} m \underline{C}^{*}\right. \\
m A^{*} m T^{*} m A^{*} m A^{*} m T^{*} m G^{*} m \underline{C}^{*} m T^{*} m G^{*} m G\end{array}$ & 175200 & 7956.3 & 7958.9 \\
\hline A-8 & $\begin{array}{l}\operatorname{ct}(P S)-m T^{*} m \underline{C}^{*} m A^{*} m C^{*} m T^{*} m T^{*} m T^{*} m \underline{C}^{*} \\
m A^{*} m T^{*} m A^{*} m A^{*} m T^{*} m G^{*} m \underline{C}^{*} m T^{*} m G^{*} m G\end{array}$ & 175200 & 7627.7 & 7627.1 \\
\hline B-1 & $\operatorname{ct}(P O)-$ GCCCAAGCUGGCAUCCGUCA & 180200 & 6505.8 & 6503.8 \\
\hline B-2 & $\operatorname{ct}(P O)-G^{*} C^{*} C^{*} C^{*} A^{*} A^{*} G^{*} C^{*} U^{*} G^{*} G^{*} C^{*} A^{*} U^{*} C^{*} C^{*} G^{*} U^{*} C^{*} A$ & 180200 & 7106.4 & 7107.3 \\
\hline B-3 & $\begin{array}{c}\operatorname{ct}(P O)- \\
m G m C m C m C m A m A m G m C m U m G m G m C m A m U m C m C m G m U m C m A\end{array}$ & 185900 & 6811.0 & 6813.4 \\
\hline B-4 & $\begin{array}{c}\operatorname{ct}(P O)-m G^{*} m C^{*} m C^{*} m C^{*} m A^{*} m A^{*} m G^{*} m C^{*} m U^{*} m G^{*} \\
m G^{*} m C^{*} m A^{*} m U^{*} m C^{*} m C^{*} m G^{*} m U^{*} m C^{*} m A\end{array}$ & 185900 & 7411.5 & 7415.3 \\
\hline B-5 & $\begin{array}{c}\operatorname{ct}(P O)-m G^{*} m C^{*} m C^{*} m C^{*} m A^{*} m A m G m C m U m G \\
m G m C m A m U m C^{*} m C^{*} m G^{*} m U^{*} m C^{*} m A\end{array}$ & 185900 & 7155.4 & 7154.5 \\
\hline B-6 & $\begin{array}{l}\operatorname{ct}(P S)-m G^{*} m C^{*} m C^{*} m C^{*} m A^{*} m A m G m C m U m G \\
m G m C m A m U m C^{*} m C^{*} m G^{*} m U^{*} m C^{*} m A\end{array}$ & 185900 & 7139.4 & 7138.7 \\
\hline
\end{tabular}




\begin{tabular}{|c|c|c|c|c|}
\hline B-7 & $\begin{array}{c}\mathrm{ct}(\text { click })-m G^{*} m C^{*} m C^{*} m C^{*} m A^{*} m A^{*} m G^{*} m C^{*} m U^{*} m G^{*} \\
m G^{*} m C^{*} m A^{*} m U^{*} m C^{*} m C^{*} m G^{*} m U^{*} m C^{*} m A\end{array}$ & 185900 & 7756.1 & 7759.7 \\
\hline $\mathrm{C}-1$ & 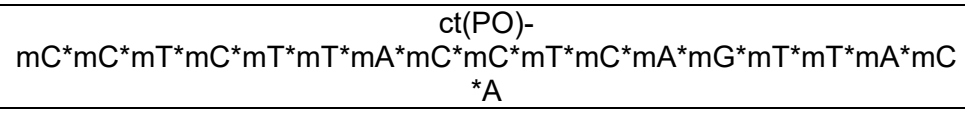 & 169700 & 6583.8 & 6582.5 \\
\hline C-2 & $\operatorname{ct}(P O)-m C^{*} C^{*} m T^{*} C^{*} m T^{*} T^{*} m A^{*} C^{*} m C^{*} T^{*} m C^{*} A^{*} m G^{*} T^{*} m T^{*} A^{*} m C^{*} A$ & 162500 & 6313.6 & 6312.3 \\
\hline C-3 & $\operatorname{ct}(P O)-m C^{*} m C^{*} T^{*} C^{*} m T^{*} m T^{*} A^{*} C^{*} m C^{*} m T^{*} C^{*} A^{*} m G^{*} m T^{*} T^{*} A^{*} m C^{*} A$ & 164000 & 6313.6 & 6314.7 \\
\hline C-4 & $\operatorname{ct}(P O)-m C^{*} m C^{*} m T^{*} C^{*} T^{*} T^{*} m A^{*} m C^{*} m C^{*} T^{*} C^{*} A^{*} m G^{*} m T^{*} m T^{*} A^{*} C^{*} A$ & 162300 & 6313.6 & $\begin{array}{c}6335.2 \\
(+\mathrm{Na}) \\
6352.9(+\mathrm{K}) \\
\end{array}$ \\
\hline C-5 & $\operatorname{ct}(P O)-m C^{*} m C^{*} m T^{*} m C^{*} m T^{*} T^{*} A^{*} C^{*} C^{*} T^{*} C^{*} A^{*} G^{*} m T^{*} m T^{*} m A^{*} m C^{*} m A$ & 164500 & 6343.6 & 6344.8 \\
\hline C-6 & $\begin{array}{c}\mathrm{ct}(\mathrm{PO})- \\
m C^{*} m C^{*} m T^{*} m C^{*} m T^{*} m T^{*} m A^{*} m C^{*} m C^{*} m T^{*} m C^{*} m A^{*} m G^{*} m T^{*} m T^{*} m A^{*} \\
m C^{*} A\end{array}$ & 169800 & 7486.1 & 7486.5 \\
\hline C-7 & $\operatorname{ct}(P O)-m C^{*} C^{*} m T^{*} C^{*} m T^{*} T^{*} m A^{*} C^{*} m C^{*} T^{*} m C^{*} A^{*} m G^{*} T^{*} m T^{*} A^{*} m C^{*} A$ & 166400 & 6851.3 & 6852.3 \\
\hline C-8 & $\operatorname{ct}(P O)-m C^{*} m C^{*} T^{*} C^{*} m T^{*} m T^{*} A^{*} C^{*} m C^{*} m T^{*} C^{*} A^{*} m G^{*} m T^{*} T^{*} A^{*} m C^{*} A$ & 166600 & 6851.3 & 6852.6 \\
\hline C-9 & ct(PO)- $m C^{*} m C^{*} m T^{*} C^{*} T^{*} T^{*} m A^{*} m C^{*} m C^{*} T^{*} C^{*} A^{*} m G^{*} m T^{*} m T^{*} A^{*} C^{*} A$ & 166300 & 6851.3 & 6852.3 \\
\hline C-10 & $\operatorname{ct}(P O)-m C^{*} m C^{*} m T^{*} m C^{*} m T^{*} T^{*} A^{*} C^{*} C^{*} T^{*} C^{*} A^{*} G^{*} m T^{*} m T^{*} m A^{*} m C^{*} m A$ & 167200 & 6850.3 & 6853.9 \\
\hline C-11 & ct(PO)- C\#C\#T\#C\#T\#T\#A\#C\#C\#T\#C\#A\#G\#T\#T\#A\#C\#A & 181860 & 6602.8 & 6604.1 \\
\hline C-12 & 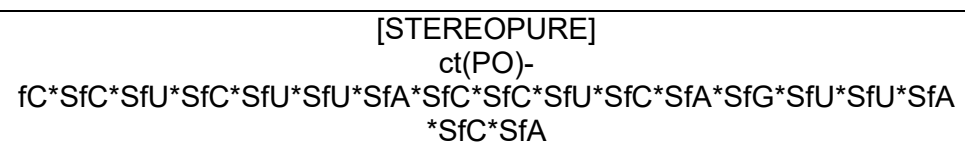 & 169700 & 6367.2 & 6365.5 \\
\hline C-13 & 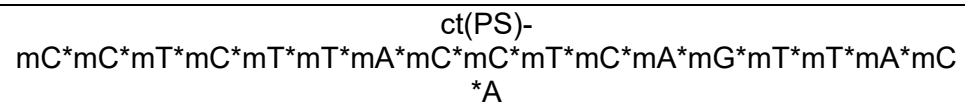 & 169700 & 6599.9 & 6598.9 \\
\hline C-14 & 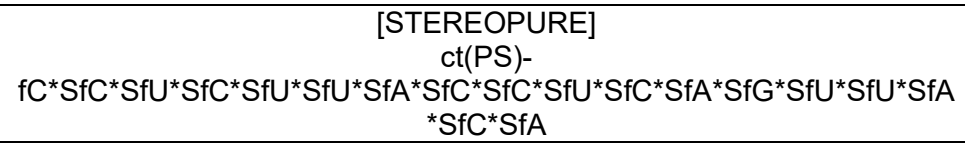 & 169700 & 6383.3 & 6382.5 \\
\hline C-15 & ct(click)-C\#C\#T\#C\#T\#T\#A\#C\#C\#T\#C\#A\#G\#T\#T\#A\#C\#A & 181860 & 6804.5 & 6806.6 \\
\hline \multirow{2}{*}{$\mathrm{D}-1$} & $\begin{array}{l}\text { Sense: fUmCfC mUfAmUfGmAfCmUfGmUfAmGfAmUfUmU fUmAfU } \\
\text { [TEG-Chol] }\end{array}$ & 213700 & 7515.1 & 7515.3 \\
\hline & $\begin{array}{l}\text { Antisense: [Phos]mAfUmAfAmAfAmUfCmUfAmCfAmGfU } \\
\text { mCfAmUfAmGfGmA*fA*mU }\end{array}$ & 251400 & 7649.9 & 7650.4 \\
\hline \multirow{2}{*}{$\mathrm{D}-2$} & $\begin{array}{l}\text { Sense: ct(PO)- fUmCfC mUfAmUfGmAfCmUfGmUfAmGfAmUfUmU } \\
\text { fUmAfU [TEG-Chol] }\end{array}$ & 213700 & 8000.0 & 8000.9 \\
\hline & $\begin{array}{c}\text { Antisense: [Phos]mAfUmAfAmAfAmUfCmUfAmCfAmGfU } \\
\text { mCfAmUfAmGfGmA*fA*mU }\end{array}$ & 251400 & 7649.8 & 7651.1 \\
\hline \multirow{2}{*}{ D-3 } & $\begin{array}{l}\text { Sense: fUmCfC mUfAmUfGmAfCmUfGmUfAmGfAmUfUmU fUmAfU } \\
\text { [TEG-Chol] }\end{array}$ & 213700 & 7515.1 & 7516.3 \\
\hline & $\begin{array}{c}\text { Antisense: [Phos]mAfUmAfAmAfAmUfCmUfAmCfAmGfU } \\
\text { mCfAmUfAmGfGmA*A*mU -ct(PO) }\end{array}$ & 251400 & 8136.0 & 8136.0 \\
\hline \multirow{2}{*}{$\mathrm{D}-4$} & $\begin{array}{l}\text { Sense: ct(PO)- fUmCfC mUfAmUfGmAfCmUfGmUfAmGfAmUfUmU } \\
\text { fUmAfU }\end{array}$ & 213700 & 7300.1 & 7301.3 \\
\hline & $\begin{array}{c}\text { Antisense: [Phos]mAfUmAfAmAfAmUfCmUfAmCfAmGfU } \\
\text { mCfAmUfAmGfGmA*f**mU }\end{array}$ & 251400 & 7649.8 & 7650.8 \\
\hline
\end{tabular}




\begin{tabular}{|c|c|c|c|c|}
\hline \multirow{2}{*}{ D-5 } & $\begin{array}{l}\text { Sense: fU*mC*fCmUfAmUfGmAfCmUfGmUfAmGfA mUfUmUfUmAfU } \\
\text { [TEG-Chol] }\end{array}$ & 213700 & 7547.2 & 7547.5 \\
\hline & $\begin{array}{c}\text { Antisense: [Phos]mA*fU*mAfAmAfAmUfCmUfAmCfAmGfU } \\
\text { mCfAmUfAmGfGmA* } A^{*} m U\end{array}$ & 251400 & 7682.0 & 7682.5 \\
\hline \multirow{2}{*}{ D-6 } & $\begin{array}{l}\text { Sense: ct(PO)- fU*mC*fCmUfAmUfGmAfCmUfGmUfAmGfAmUfUmU } \\
\text { fUmAfU [TEG-Chol] }\end{array}$ & 213700 & 8032.1 & 8033.1 \\
\hline & $\begin{array}{l}\text { Antisense: [Phos]mA*fU*mAfAmAfAmUfCmUfAmCfAmGfU } \\
\text { mCfAmUfAmGfGmA* }{ }^{*} A^{*} m U\end{array}$ & 251400 & 7682.0 & 7682.9 \\
\hline \multirow{2}{*}{$\mathrm{D}-7$} & $\begin{array}{l}\text { Sense: fU*mC*fmUfAmUfGmAfC mUfGmU fAmGfAmUfUmU fUmAfU } \\
\text { [TEG-Chol] }\end{array}$ & 213700 & 7547.2 & 7548.1 \\
\hline & $\begin{array}{l}\text { Antisense: [Phos]mA*fU*mAfAmAfAmUfCmUfAmCfAmGfU } \\
\text { mCfAmUfAmGfGmA*fA*mU -ct(PO) }\end{array}$ & 251400 & 8168.2 & 8168.0 \\
\hline \multirow{2}{*}{ D-8 } & $\begin{array}{l}\text { Sense: fU*mC*fCmUfAmUfGmAfCmUfGmUfAmGfA mUfUmU fU*mA*fU } \\
\text { [TEG-Chol] }\end{array}$ & 213700 & 7579.4 & 7579.7 \\
\hline & $\begin{array}{l}\text { Antisense: [Phos]mA*fU*mAfAmA fAmUfC mUfAmCfAmGfU } \\
m \mathrm{mff}^{*} m U^{*} f A^{*} m G^{*} f G^{*} m A^{*} f A^{*} m U\end{array}$ & 251400 & 7762.4 & 7761.7 \\
\hline \multirow{2}{*}{ D-9 } & $\begin{array}{l}\text { Sense: ct(PO)- fU*mC*fCmUfAmUfGmAfCmUfGmUfAmGfA mUfUmU } \\
\text { fU*mA*fU [TEG-Chol] }\end{array}$ & 213700 & 8064.2 & 8065.4 \\
\hline & $\begin{array}{l}\text { Antisense: [Phos]mA*fU*mAfAmA fAmUfC mUfAmCfAmGfU } \\
m \mathrm{mff}^{*} m U^{\star} f A^{*} m G^{*} \mathrm{fG}^{*} m A^{*} f A^{*} m U\end{array}$ & 251400 & 7762.3 & 7763.4 \\
\hline \multirow{2}{*}{ D-10 } & $\begin{array}{l}\text { Sense: fU*mC*fCmUfAmUfGmAfCmUfGmUfAmGfA mUfUmU fU*mA*fU } \\
\text { [TEG-Chol] }\end{array}$ & 213700 & 7579.4 & 7580.3 \\
\hline & $\begin{array}{c}\text { Antisense: [Phos]mA*fU*mAfAmA fAmUfC mUfAmCfAmGfU } \\
m C f A^{*} m U^{*} f A^{*} m G^{*} f G^{*} m A^{*} f A^{*} m U-\operatorname{ct}(P O)\end{array}$ & 251400 & 8248.5 & 8247.9 \\
\hline \multirow{2}{*}{ D-11 } & $\begin{array}{l}\text { Sense: ct-fU*mC*fCmUfAmUfGmAfCmUfGmUfAmGfA mUfUmU } \\
\qquad U^{*} m A^{*} f U\end{array}$ & 213700 & 7364.4 & 7365.4 \\
\hline & $\begin{array}{l}\text { Antisense: [Phos]mA*fU*mAfAmA fAmUfC mUfAmCfAmGfU } \\
m C f A^{*} m U^{*} f A^{*} m G^{*} f G^{*} m A^{*} f A^{*} m U\end{array}$ & 251400 & 7762.3 & 7763.1 \\
\hline$E-1$ & $\begin{array}{c}\operatorname{ct}(P O)- \\
m U^{*} m G m \underline{C} m \underline{C} m A^{*} G^{*} G^{*} C^{*} T^{*} G^{*} G^{*} T^{*} T^{*} A^{*} T^{*} m G^{*} m A^{*} m C^{*} m U^{*} m C\end{array}$ & 183900 & 7298.4 & 7297.0 \\
\hline $\mathrm{E}-2$ & 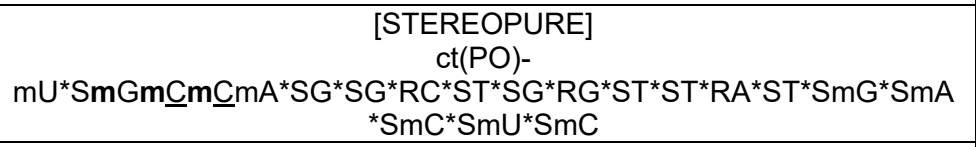 & 183900 & 7298.4 & 7297.3 \\
\hline$E-5$ & $\begin{array}{c}\operatorname{ct}(P S)- \\
m U^{*} m G m \underline{C} m \underline{C} m A^{*} G^{*} G^{*} C^{*} T^{*} G^{*} G^{*} T^{*} T^{*} A^{*} T^{*} m G^{*} m A^{*} m C^{*} m U^{*} m C\end{array}$ & 183900 & 7314.4 & 7313.5 \\
\hline E-6 & 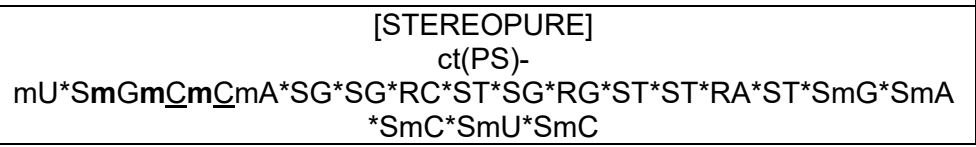 & 183900 & 7314.4 & 7313.7 \\
\hline
\end{tabular}


ct- $\mathrm{COOH}$

Molecular Weight: 323.81

狖ONOH

Molecular Weight: 636.19<smiles></smiles>

ct-DBCO

Molecular Weight: 511.06<smiles>O=C(CCC(=O)N1Cc2ccccc2C#Cc2ccccc21)NCCOCCOCCCCCCCl</smiles>

ct-W

Molecular Weight: 509.04<smiles>NC(=O)C(CC1=CNCC1)NC(=O)CCC(=O)NCCOCCOCCCCCCCl</smiles>

$\operatorname{ct}(P O)$ Linker<smiles>C[13CH2]OP(=O)([O-])OCCCCCCNC(=O)CCC(=O)NCCOCCOCCCCCCl</smiles>

ct(PO)-click linker<smiles></smiles>

ct(PS) Linker<smiles>C[13CH2]OP(=O)([S-])OCCCCCCNC(=O)CCC(=O)NCCOCCOCCCCCCCl</smiles>

SI Figure 1. Chemical structures. Structures and molecular weights of chloroalkane tag reagents and chloroalkane-tagged small molecules, and structures of chloroalkane tags shown with $5^{\prime}$ phosphodiester or phosphorothioate. 
A.

\begin{tabular}{|ccccc|}
\hline Name & Backbone & Sugar & $\mathrm{CP}_{50}(\mu \mathrm{M})$ \\
\hline- & B-1 & $\mathrm{PO}$ & $2 \mathrm{H}$ & $4.2 \pm 1.1$ \\
\hline- & B-2 & $\mathrm{PS}$ & $2 \mathrm{H}$ & $2.6 \pm 0.4$ \\
\hline- & B-3 & $\mathrm{PO}$ & $2 \mathrm{OMe}$ & $11.9 \pm 5.3$ \\
\hline- & B-4 & $\mathrm{PS}$ & $2 \mathrm{OMe}$ & $0.6 \pm 0.04$ \\
\hline- & ct-W & $\mathrm{n} / \mathrm{a}$ & $\mathrm{n} / \mathrm{a}$ & $0.03 \pm 0.01$ \\
\hline
\end{tabular}

B.

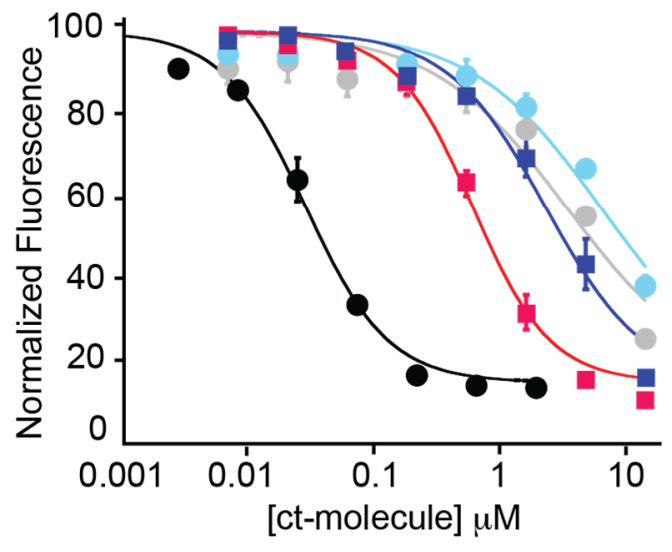

C.

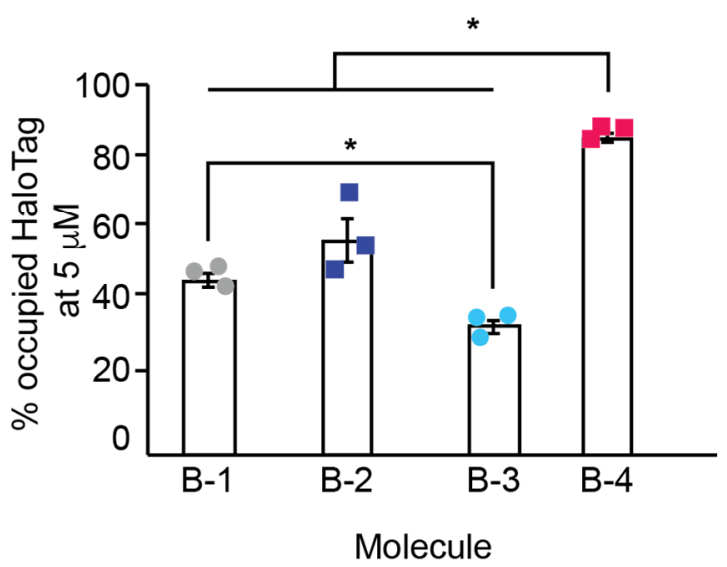

SI Figure 2. Application of CAPA to ASOs with sequences derived from the drug alicaforsen. A. Table of compounds with $\mathrm{CP}_{50}$ values for four compounds with sequence $B$ (GCCCAAGCUGGCAUCCGUCA) with different combinations of commonly used backbone and sugar modifications. Phosphodiester backbones are denoted PO, phosphorothioate backbones are denoted PS, deoxyribose sugars are denoted $2 \mathrm{H}$, sugars modified with 2'-O-methyl modifications are denoted 2OMe, and sugars modified with 2'-O-methoxyethyl modifications are denoted 2MOE. B. Dose-dependent CAPA curves. Cells were incubated with the indicated concentration of ct-ASO for $4 \mathrm{~h}$ in serum-free media. C. Percent occupied HaloTag at $5 \mu \mathrm{M}$ of applied ct-ASO was calculated from the CAPA signal of each compound. Compound B-4 is significantly different from compounds B-1, B-2, and B-3 and compounds B1 and B-3 are significantly different from one another (asterisks denote $p$ values $<0.05$ by two-tailed t-test). ct-W, shown in SI Fig 1, is a small molecule included as a positive control. Error bars represent the standard error from three independent trials. $\mathrm{CP}_{50}$ values are the averages and standard errors calculated from three independent curve fits. CP50 values are also listed in SI Table 2. 


\begin{tabular}{|ccccc|}
\hline Name & Sequence & Backbone & Sugar \\
\hline$\bullet$ & A-1 & A & PO & $2 \mathrm{H}$ \\
\hline$\leftarrow$ & A-2 & A & PS & $2 \mathrm{H}$ \\
\hline$\leftarrow$ & A-3 & A & PO & $2 \mathrm{OMe}$ \\
\hline$\leftarrow$ & A-4 & A & PS & $2 \mathrm{OMe}$ \\
\hline- & B-1 & B & PO & $2 \mathrm{H}$ \\
\hline$\bullet$ & B-2 & B & PS & $2 \mathrm{H}$ \\
\hline- & B-3 & B & PO & $2 \mathrm{OMe}$ \\
\hline$\bullet$ & B-4 & B & PS & $2 \mathrm{OMe}$ \\
\hline
\end{tabular}

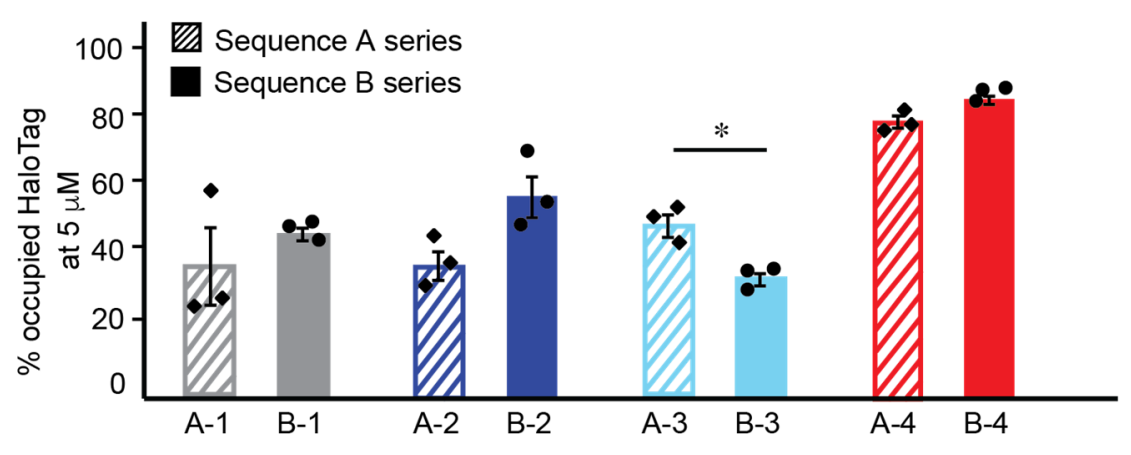

Backbone and sugar modification

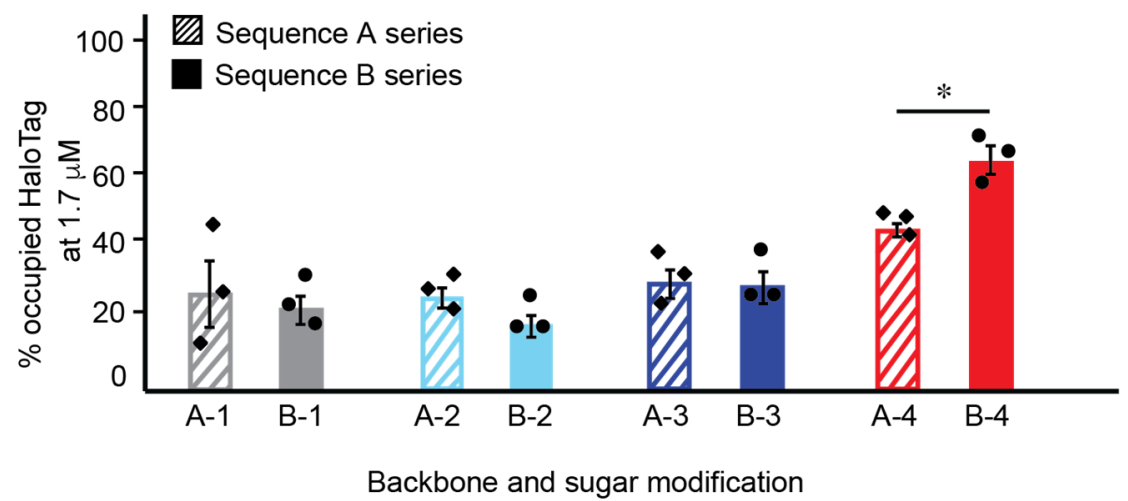

SI Figure 3. Effects of sequence and length on cytosolic penetration. Cells were incubated with the indicated concentration of ct-ASO for $4 \mathrm{~h}$ in serum-free media. Percent occupied HaloTag at $5 \mu \mathrm{M}$ and at $1.7 \mu \mathrm{M}$ of applied ct-ASO were calculated from the CAPA signal. The percent occupied HaloTag at $5 \mu \mathrm{M}$ of applied ct-RNA is significantly larger for sequence $A$ than sequence $B$ for the PO-2OMe modified compounds A-3 and B-3 ( $p$ value $<0.05$ ). The percent occupied HaloTag at $5 \mu \mathrm{M}$ of applied ct-RNA is nearsignificantly smaller for sequence $A$ than sequence $B$ for the PS-2H compounds, A-2 and B-2 ( $p$ value 0.06 ), and the PS-2OMe modified compounds, A-4 and B-4 ( $p$ value 0.07 ). The percent occupied HaloTag at $1.7 \mu \mathrm{M}$ of applied ct-RNA is significantly larger for sequence $B$ than sequence $A$ for the PS-2OMe modified compounds, A-4 and B-4 ( $p$ value <0.05). Asterisks denote $p$ values below 0.05 as determined using a two-tailed t-test. ct-W, shown in SI Fig 1, is a small molecule included as a positive control. Error bars represent the standard error from three independent trials. $\mathrm{CP}_{50}$ values are the averages and standard errors calculated from three independent curve fits. 
A.
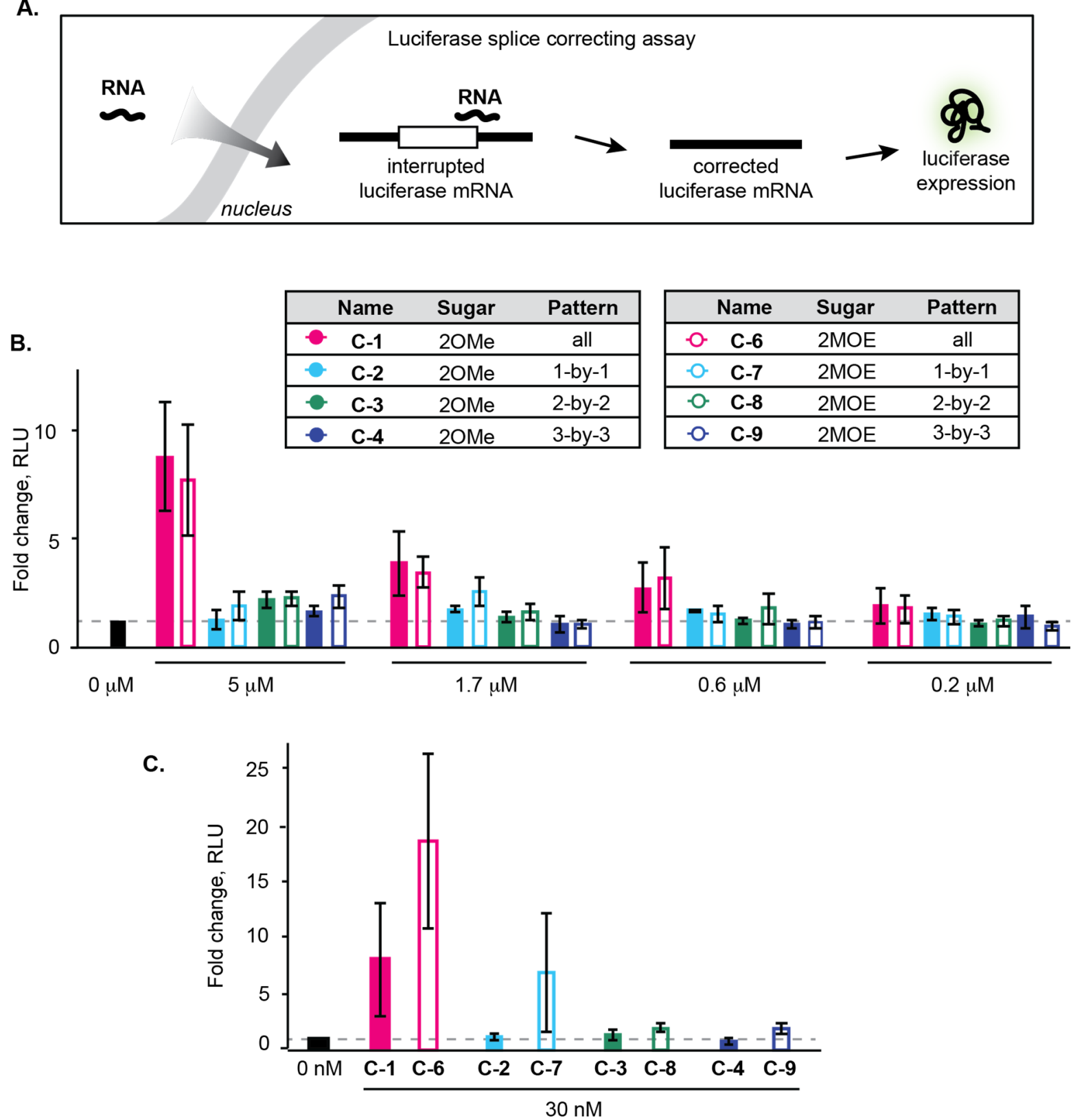

SI Figure 4. Effects of 2' modification patterning on activity of splice-correcting ASOs. A. Schematic of luciferase splice-correcting assay. HeLa cells stably expressing an interrupted luciferase mRNA transcript are incubated with oligonucleotides designed to correct mRNA splicing (sequence $\mathrm{C}$ ). The resulting increase in the expression of functional, full-length luciferase is measured using a luciferase substrate and a plate reader. B. Luciferase splice-correction activity calculated as fold change RLU at 30 $\mathrm{nM}$ of each oligonucleotide complexed with lipofectamine. Cells were incubated with $30 \mathrm{nM}$ ct-RNA complexed with lipofectamine for $4 \mathrm{~h}$, and then media was replaced. The cells were then incubated for 48 $\mathrm{h}$ before detection. C. Luciferase splice-correction activity calculated as fold change RLU at $5 \mu \mathrm{M}$ for oligonucleotides internalized through gymnotic uptake. Cells were incubated with the indicated concentration of ct-RNA for $48 \mathrm{~h}$ in serum-free media. Error bars represent the standard error from three independent trials. 


\begin{tabular}{|c|c|c|c|c|}
\hline Name & ct- location & \# PS & cholesterol & $\mathrm{EC}_{50}(\mathrm{nM})$ \\
\hline$\prec D-1$ & --- & 2 & $\sqrt{ }$ & $2.1 \pm 0.6$ \\
\hline$\simeq \mathrm{D}-2$ & sense & 2 & $\sqrt{ }$ & $1.9 \pm 1.1$ \\
\hline- D-3 & antisense & 2 & $\sqrt{ }$ & $3.0 \pm 0.5$ \\
\hline D-4 & sense & 2 & --- & $>100$ \\
\hline$\prec D-5$ & --- & 6 & $\sqrt{ }$ & $2.3 \pm 0.3$ \\
\hline $\pm D-6$ & sense & 6 & $\sqrt{ }$ & $2.0 \pm 0.4$ \\
\hline - D-7 & antisense & 6 & $\sqrt{ }$ & $5.0 \pm 1.5$ \\
\hline$\checkmark D-8$ & --- & 12 & $\sqrt{ }$ & $6.2 \pm 0.1$ \\
\hline$\neq D-9$ & sense & 12 & $\sqrt{ }$ & $17.5 \pm 0.6$ \\
\hline D-10 & antisense & 12 & $\sqrt{ }$ & $29.8 \pm 17.0$ \\
\hline$+\mathrm{D}-11$ & sense & 12 & -- & $>100$ \\
\hline
\end{tabular}

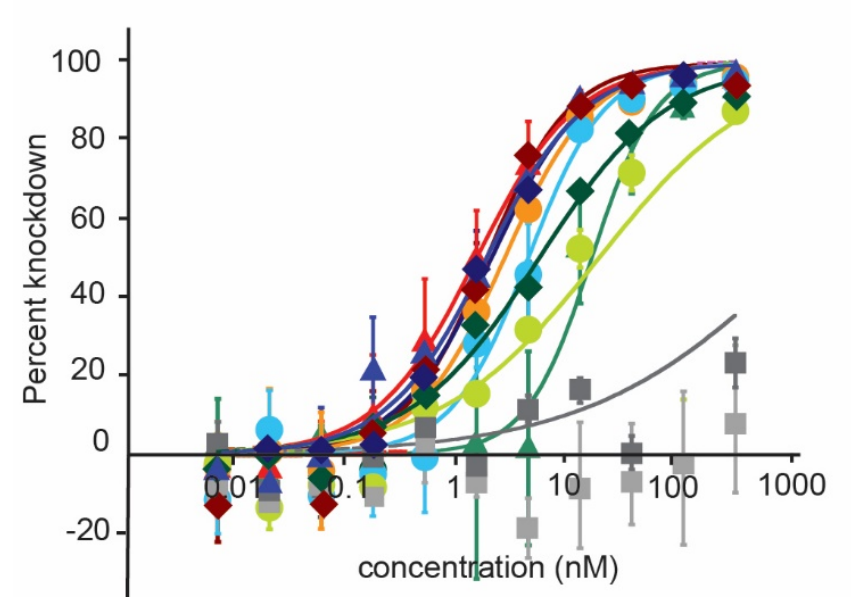

SI Figure 5. Knockdown activity of siRNAs. Dose-dependent activity curves and $\mathrm{EC}_{50}$ values for $H P R T$ knockdown. Cells were incubated with the indicated concentration of ct-siRNA for $72 \mathrm{~h}$ in serum-free media. Error bars represent the standard error from three independent measurements. $\mathrm{EC}_{50}$ values represent the averages and standard errors calculated from three independent curve fits. 


\begin{tabular}{|c|c|c|c|c|}
\hline Name & ct- location & \# PS & cholesterol & $\mathrm{EC}_{50}(\mathrm{nM})$ \\
\hline$\diamond \mathrm{D}-1$ & --- & 2 & $\sqrt{ }$ & $0.003 \pm 0.001$ \\
\hline$\therefore \mathrm{D}-2$ & sense & 2 & $\checkmark$ & $0.013 \pm 0.009$ \\
\hline$-D-3$ & antisense & 2 & $\sqrt{ }$ & $0.009 \pm 0.0001$ \\
\hline$-\mathrm{D}-4$ & sense & 2 & --- & $0.0004 \pm 0.0004$ \\
\hline$\star D-5$ & --- & 6 & $\sqrt{ }$ & $0.009 \pm 0.005$ \\
\hline$\pm \mathrm{D}-6$ & sense & 6 & $\sqrt{ }$ & $0.007 \pm 0.003$ \\
\hline$\rightarrow D-7$ & antisense & 6 & $\sqrt{ }$ & $0.008 \pm 0.001$ \\
\hline$\sim D-8$ & -- & 12 & $\checkmark$ & $0.030 \pm 0.003$ \\
\hline$\simeq \mathrm{D}-9$ & sense & 12 & $\sqrt{ }$ & $0.15 \pm 0.12$ \\
\hline D-10 & antisense & 12 & $\sqrt{ }$ & $0.022 \pm 0.001$ \\
\hline D-11 & sense & 12 & -- & $0.013 \pm 0.012$ \\
\hline
\end{tabular}

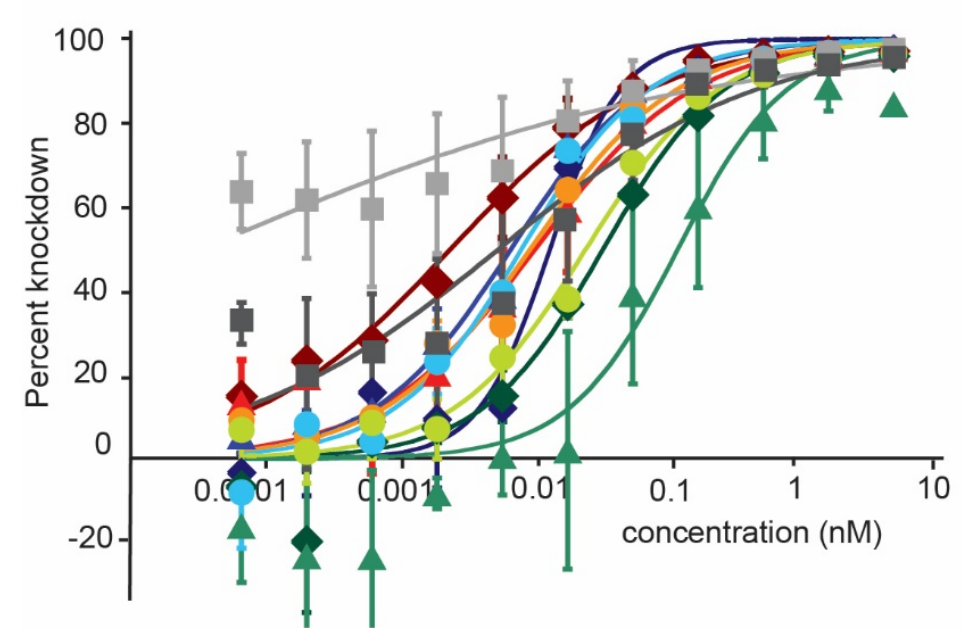

SI Figure 6. Knockdown activity of siRNAs after transfection using lipofectamine. Dose-dependent activity curves and $\mathrm{EC}_{50}$ values for HPRT mRNA knockdown after transfection-facilitated delivery. siRNAs were complexed with Lipofectamine RNAiMax (ThermoFisher), and then incubated with cells for $24 \mathrm{~h}$ in serum-free media before measuring knockdown using RT-qPCR. Error bars represent the standard error from three independent measurements. $\mathrm{EC}_{50}$ values represent the averages and standard errors calculated from three independent curve fits. 


\begin{tabular}{|ccccc|}
\hline Name & ct- location & \# of PS & cholesterol & $\operatorname{Tm}\left({ }^{\circ} \mathbf{C}\right)$ \\
\hline D-1 & --- & 2 & $\checkmark$ & $70.48 \pm 0.07$ \\
\hline D-2 & sense & 2 & $\checkmark$ & $70.51 \pm 0.03$ \\
\hline D-3 & antisense & 2 & $\checkmark$ & $69.79 \pm 0.03$ \\
\hline D-4 & sense & 2 & --- & $69.45 \pm 0.12$ \\
\hline D-5 & --- & 6 & $\checkmark$ & $70.13 \pm 0.03$ \\
\hline D-6 & sense & 6 & $\checkmark$ & $69.82 \pm 0.08$ \\
\hline D-7 & antisense & 6 & $\checkmark$ & $70.44 \pm 0.00$ \\
\hline D-8 & --- & 12 & $\checkmark$ & $68.59 \pm 0.03$ \\
\hline D-9 & sense & 12 & $\checkmark$ & $68.93 \pm 0.00$ \\
\hline D-10 & antisense & 12 & $\checkmark$ & $69.03 \pm 0.03$ \\
\hline D-11 & sense & 12 & --- & $67.04 \pm 0.07$ \\
\hline
\end{tabular}

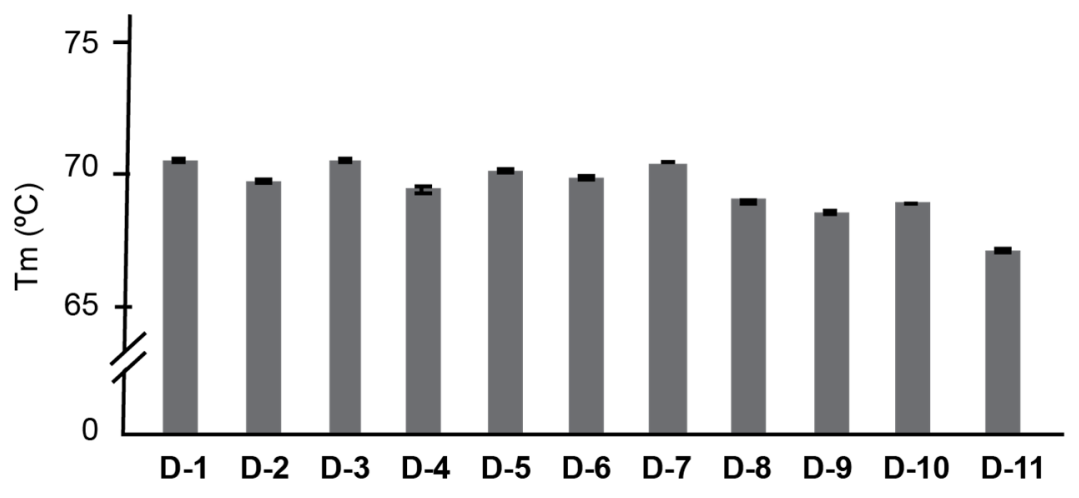

SI Figure 7. Melting temperatures $\left(T_{m}\right)$ of siRNA duplexes. The $T_{m}$ of each siRNA duplex was measured as described in Methods. Error bars represent the standard error from three independent measurements. 


\begin{tabular}{|c|c|c|c|c|}
\hline Name & ct- location & \# of PS & cholesterol & $\begin{array}{c}\text { loaded antisense } \\
(\mathrm{pg} / \mu \mathrm{L})\end{array}$ \\
\hline D-1 & -- & 2 & $\sqrt{ }$ & $0.20 \pm 0.03$ \\
\hline D-2 & sense & 2 & $\sqrt{ }$ & $0.17 \pm 0.03$ \\
\hline D-3 & antisense & 2 & $\sqrt{ }$ & $0.10 \pm 0.02$ \\
\hline$D-4$ & sense & 2 & --- & $0.18 \pm 0.02$ \\
\hline D-5 & --- & 6 & $\sqrt{ }$ & $0.16 \pm 0.01$ \\
\hline D-6 & sense & 6 & $\sqrt{ }$ & $0.13 \pm 0.01$ \\
\hline D-7 & antisense & 6 & $\sqrt{ }$ & $0.15 \pm 0.01$ \\
\hline D-8 & -- & 12 & $\sqrt{ }$ & $0.13 \pm 0.02$ \\
\hline D-9 & sense & 12 & $\sqrt{ }$ & $0.16 \pm 0.01$ \\
\hline$D-10$ & antisense & 12 & $\sqrt{ }$ & $0.10 \pm 0.03$ \\
\hline D-11 & sense & 12 & --- & $0.14 \pm 0.02$ \\
\hline
\end{tabular}

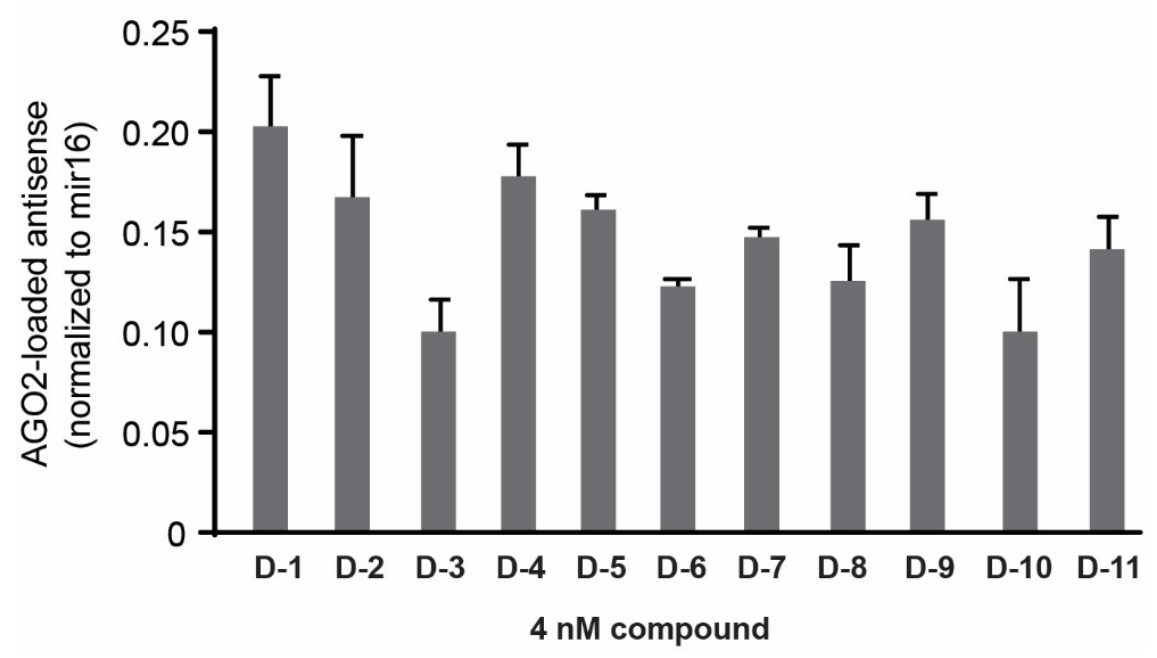

SI Figure 8. RISC loading of siRNA duplexes. The amount of antisense strand that was loaded into Ago2 for each siRNA in a cell lysate was measured as described in Methods. Error bars represent the standard error from three independent measurements. 


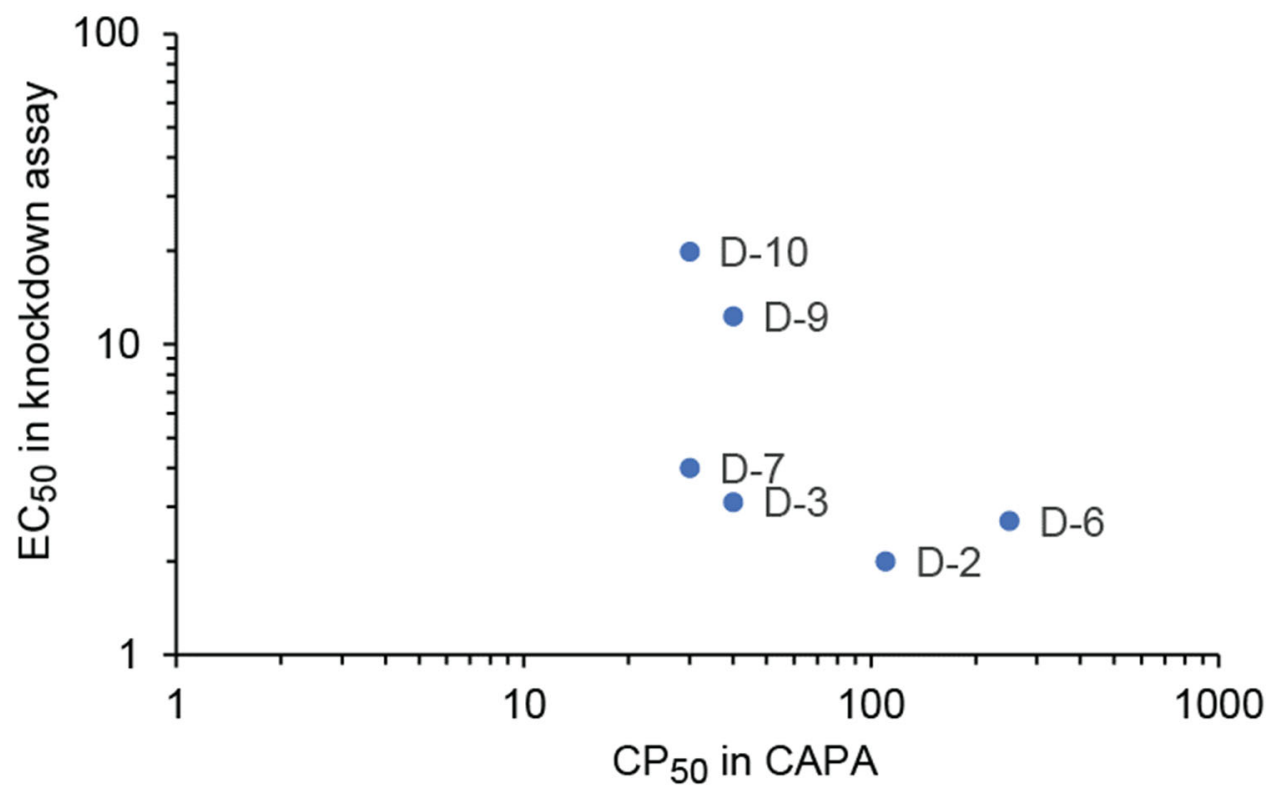

SI Figure 9. Correlation of $\mathrm{EC}_{50}$ and $\mathrm{CP}_{50}$ for selected ct-siRNAs. $\mathrm{EC}_{50}$ values from the HPRT mRNA knockdown assay and $\mathrm{CP}_{50}$ values from CAPA are plotted for all ct-siRNAs with high performance in both assays. For these highly potent siRNAs, no direct correlation was observed. 


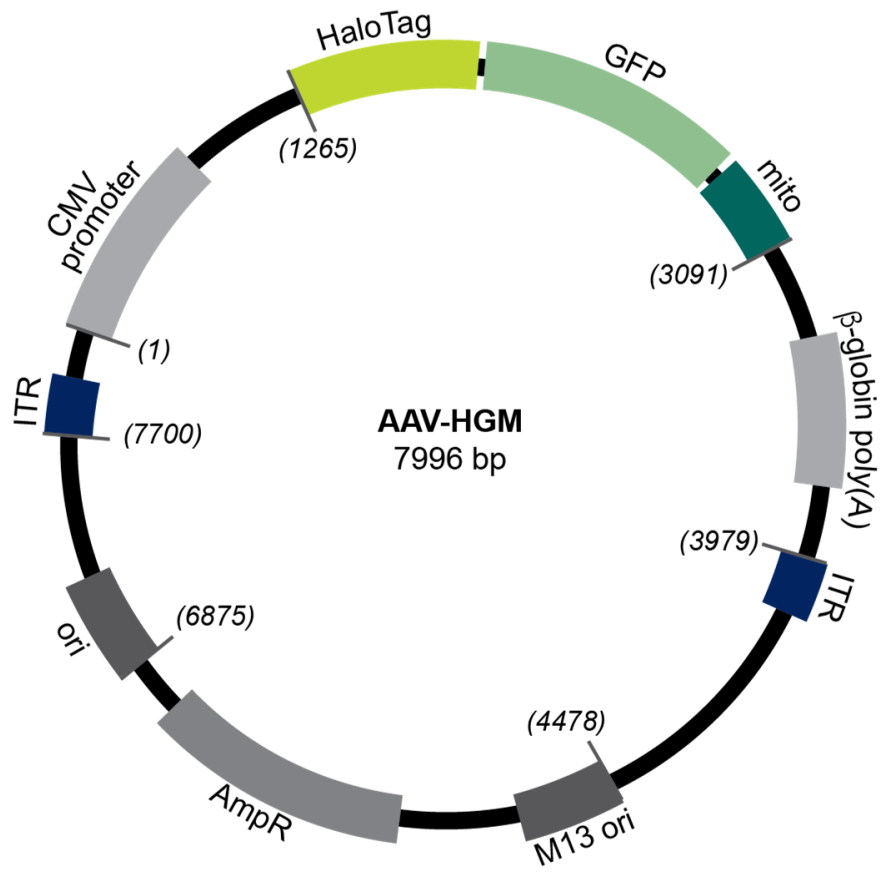

SI Figure 10. Plasmid map of key features of AAV-HGM. 
ATGGCAGAAATCGGTACTGGCTTTCCATTCGACCCCCATTATGTGGAAGTCCTGGGCGAGCGCATGCACTACGT CGATGTTGGTCCGCGCGATGGCACCCCTGTGCTGTTCCTGCACGGTAACCCGACCTCCTCCTACGTGTGGCGC AACATCATCCCGCATGTTGCACCGACCCATCGCTGCATTGCTCCAGACCTGATCGGTATGGGCAAATCCGACAA ACCAGACCTGGGTTATTTCTTCGACGACCACGTCCGCTTCATGGATGCCTTCATCGAAGCCCTGGGTCTGGAAG AGGTCGTCCTGGTCATTCACGACTGGGGCTCCGCTCTGGGTTTCCACTGGGCCAAGCGCAATCCAGAGCGCGT CAAAGGTATTGCATTTATGGAGTTCATCCGCCCTATCCCGACCTGGGACGAATGGCCAGAATTTGCCCGCGAGA CCTTCCAGGCCTTCCGCACCACCGACGTCGGCCGCAAGCTGATCATCGATCAGAACGTTTTTATCGAGGGTACG CTGCCGATGGGTGTCGTCCGCCCGCTGACTGAAGTCGAGATGGACCATTACCGCGAGCCGTTCCTGAATCCTG TTGACCGCGAGCCACTGTGGCGCTTCCCAAACGAGCTGCCAATCGCCGGTGAGCCAGCGAACATCGTCGCGCT GGTCGAAGAATACATGGACTGGCTGCACCAGTCCCCTGTCCCGAAGCTGCTGTTCTGGGGCACCCCAGGCGTT CTGATCCCACCGGCCGAAGCCGCTCGCCTGGCCAAAAGCCTGCCTAACTGCAAGGCTGTGGACATCGGCCCG GGTCTGAATCTGCTGCAAGAAGACAACCCGGACCTGATCGGCAGCGAGATCGCGCGCTGGCTGTCGACGCTCG AGATTTCCGGTTACGTAGCCACCATGGTGAGCAAGGGCGAGGAGCTGTTCACCGGGGTGGTGCCCATCCTGGT CGAGCTGGACGGCGACGTAAACGGCCACAAGTTCAGCGTGTCCGGCGAGGGCGAGGGCGATGCCACCTACGG CAAGCTGACCCTGAAGTTCATCTGCACCACCGGCAAGCTGCCCGTGCCCTGGCCCACCCTCGTGACCACCCTG ACCTACGGCGTGCAGTGCTTCAGCCGCTACCCCGACCACATGAAGCAGCACGACTTCTTCAAGTCCGCCATGC CCGAAGGCTACGTCCAGGAGCGCACCATCTTCTTCAAGGACGACGGCAACTACAAGACCCGCGCCGAGGTGAA GTTCGAGGGCGACACCCTGGTGAACCGCATCGAGCTGAAGGGCATCGACTTCAAGGAGGACGGCAACATCCTG GGGCACAAGCTGGAGTACAACTACAACAGCCACAACGTCTATATCATGGCCGACAAGCAGAAGAACGGCATCAA GGTGAACTTCAAGATCCGCCACAACATCGAGGACGGCAGCGTGCAGCTCGCCGACCACTACCAGCAGAACACC CCCATCGGCGACGGCCCCGTGCTGCTGCCCGACAACCACTACCTGAGCACCCAGTCCGCCCTGAGCAAAGACC CCAACGAGAAGCGCGATCACATGGTCCTGCTGGAGTTCGTGACCGCCGCCGGGATCACTCTCGGCATGGACGA GCTGTACAAGGGTGGTGAATCCGGTGATGCATCGGGAAGTGGAAGTGGATCTCGAGCTCAAGCTTCGAATTCAA AACTAATTGCTAAAAGTGCAGAAGACGAAAAAGCGAAGGAAGAACCAGGGAACCATAGGATCGTAATTCTTGCA ATGTTAGCTATTGGCGTGTTCTCTTTAGGGGCGCTTATCAAAATTATTCAATTAAGAAAAAATAATTAA

SI Figure 11. Sequence of the transgene open reading frame for the AAV-HGM plasmid. Bases 1265 through 3091 of the plasmid corresponding to the open reading frame encoding for the HaloTag-GFP-mito fusion. Underlined bases correspond to HaloTag-encoding region, green bases correspond to the GFPencoding region. and bold bases correspond to the peptide that localizes the fusion to the outside of the outer mitochondrial membrane. This open reading frame was inserted into an AAV-CMV-GFP vector (Addgene 67634). 


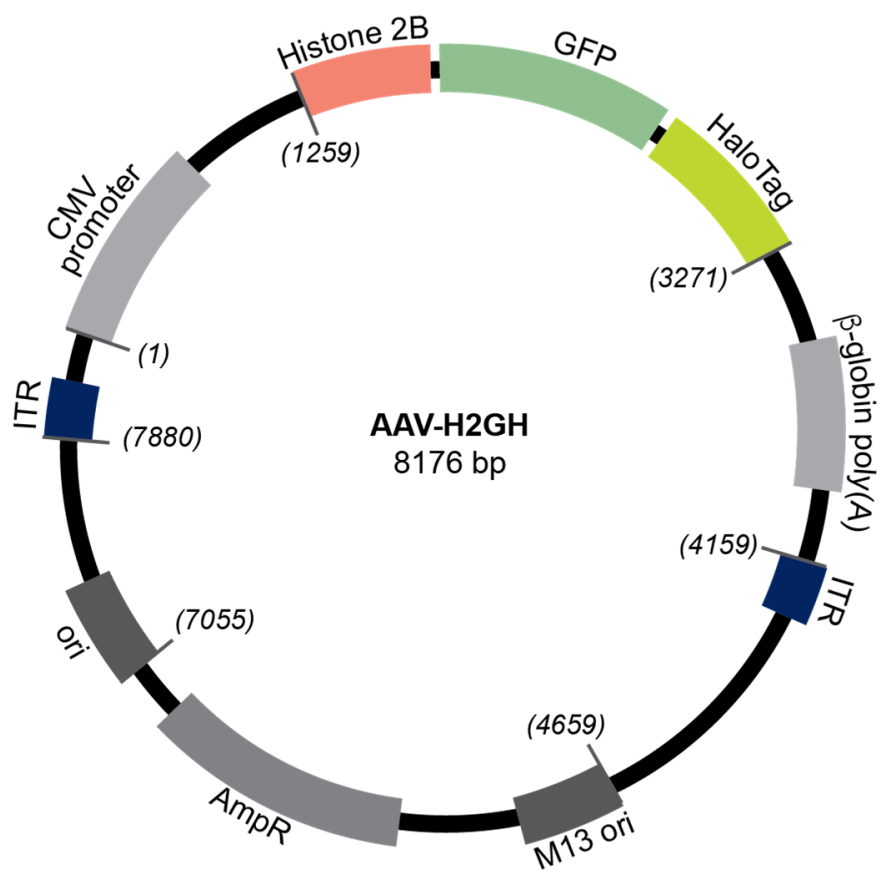

SI Figure 12. Plasmid map of key features of AAV-H2GH. 


\begin{abstract}
ATGCCAGAGCCAGCGAAGTCTGCTCCCGCCCCGAAAAAGGGCTCCAAGAAGGCGGTGACTAAGGCGCAGAA GAAAGGCGGCAAGAAGCGCAAGCGCAGCCGCAAGGAGAGCTATTCCATCTATGTGTACAAGGTTCTGAAGCA GGTCCACCCTGACACCGGCATTTCGTCCAAGGCCATGGGCATCATGAATTCGTTTGTGAACGACATTTTCGAG CGCATCGCAGGTGAGGCTTCCCGCCTGGCGCATTACAACAAGCGCTCGACCATCACCTCCAGGGAGATCCAG ACGGCCGTGCGCCTGCTGCTGCCTGGGGAGTTGGCCAAGCACGCCGTGTCCGAGGGTACTAAGGCCATCAC CAAGTACACCAGCGCTAAGGATCCACCGGTCGCCACCATGGTGAGCAAGGGCGAGGAGCTGTTCACCGGGGT GGTGCCCATCCTGGTCGAGCTGGACGGCGACGTAAACGGCCACAAGTTCAGCGTGTCCGGCGAGGGCGAGGG CGATGCCACCTACGGCAAGCTGACCCTGAAGTTCATCTGCACCACCGGCAAGCTGCCCGTGCCCTGGCCCACC CTCGTGACCACCCTGACCTACGGCGTGCAGTGCTTCAGCCGCTACCCCGACCACATGAAGCAGCACGACTTCTT CAAGTCCGCCATGCCCGAAGGCTACGTCCAGGAGCGCACCATCTTCTTCAAGGACGACGGCAACTACAAGACC CGCGCCGAGGTGAAGTTCGAGGGCGACACCCTGGTGAACCGCATCGAGCTGAAGGGCATCGACTTCAAGGAG GACGGCAACATCCTGGGGCACAAGCTGGAGTACAACTACAACAGCCACAACGTCTATATCATGGCCGACAAGCA GAAGAACGGCATCAAGGTGAACTTCAAGATCCGCCACAACATCGAGGACGGCAGCGTGCAGCTCGCCGACCAC TACCAGCAGAACACCCCCATCGGCGACGGCCCCGTGCTGCTGCCCGACAACCACTACCTGAGCACCCAGTCCG CCCTGAGCAAAGACCCCAACGAGAAGCGCGATCACATGGTCCTGCTGGAGTTCGTGACCGCCGCCGGGATCAC TCTCGGCATGGACGAGCTGTACAAGCGCGCCACCATGGCAGAAATCGGTACTGGCTTTCCATTCGACCCCCATT ATGTGGAAGTCCTGGGCGAGCGCATGCACTACGTCGATGTTGGTCCGCGCGATGGCACCCCTGTGCTGTTCCT GCACGGTAACCCGACCTCCTCCTACGTGTGGCGCAACATCATCCCGCATGTTGCACCGACCCATCGCTGCATTG CTCCAGACCTGATCGGTATGGGCAAATCCGACAAACCAGACCTGGGTTATTTCTTCGACGACCACGTCCGCTTC ATGGATGCCTTCATCGAAGCCCTGGGTCTGGAAGAGGTCGTCCTGGTCATTCACGACTGGGGCTCCGCTCTGG GTTTCCACTGGGCCAAGCGCAATCCAGAGCGCGTCAAAGGTATTGCATTTATGGAGTTCATCCGCCCTATCCCG ACCTGGGACGAATGGCCAGAATTTGCCCGCGAGACCTTCCAGGCCTTCCGCACCACCGACGTCGGCCGCAAGC TGATCATCGATCAGAACGTTTTTATCGAGGGTACGCTGCCGATGGGTGTCGTCCGCCCGCTGACTGAAGTCGAG ATGGACCATTACCGCGAGCCGTTCCTGAATCCTGTTGACCGCGAGCCACTGTGGCGCTTCCCAAACGAGCTGC CAATCGCCGGTGAGCCAGCGAACATCGTCGCGCTGGTCGAAGAATACATGGACTGGCTGCACCAGTCCCCTGT CCCGAAGCTGCTGTTCTGGGGCACCCCAGGCGTTCTGATCCCACCGGCCGAAGCCGCTCGCCTGGCCAAAAG CCTGCCTAACTGCAAGGCTGTGGACATCGGCCCGGGTCTGAATCTGCTGCAAGAAGACAACCCGGACCTGATC GGCAGCGAGATCGCGCGCTGGCTGTCGACGCTCGAGATTTCCTAA
\end{abstract}

SI Figure 13. Sequence of the transgene open reading frame for the AAV-H2GH plasmid. Bases 1259 through 3271 of the plasmid corresponding to the open reading frame encoding for the Histone2B-GFPHaloTag fusion. Underlined bases correspond to HaloTag-encoding region, green bases correspond to the GFP-encoding region, and bold bases correspond to the Histone 2B-encoding region. This open reading frame was inserted into an AAV-CMV-GFP vector (Addgene 67634). 
A.

stable HGM HeLa

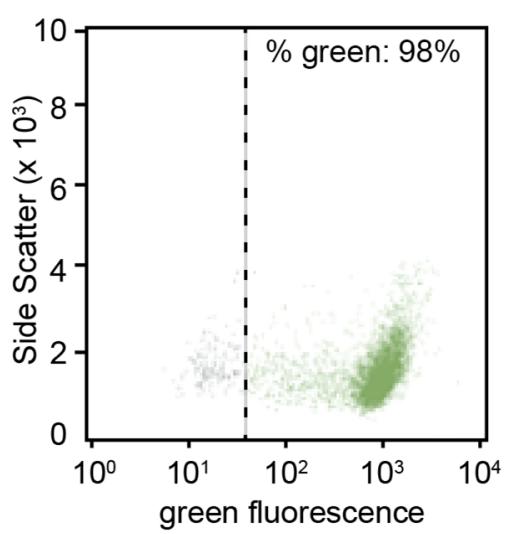

AAV-HGM-HeLa

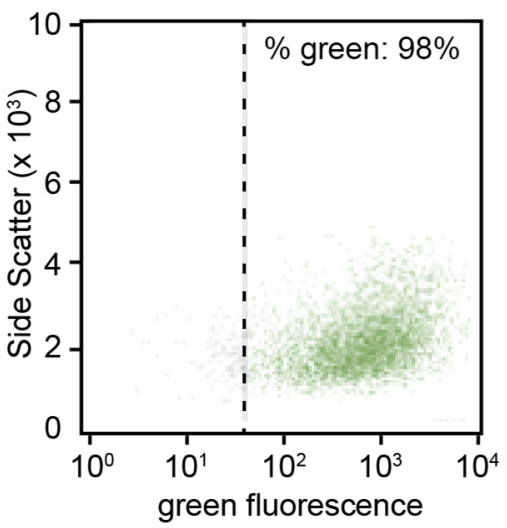

AAV-H2GH-HeLa

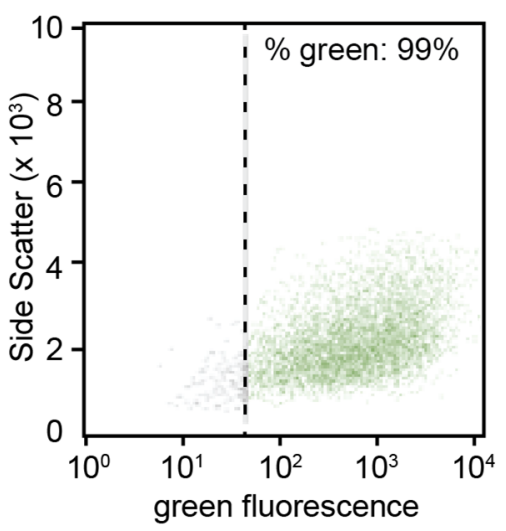

B.

stable HGM HeLa

AAV-HGM-HeLa

AAV-H2GH-HeLa
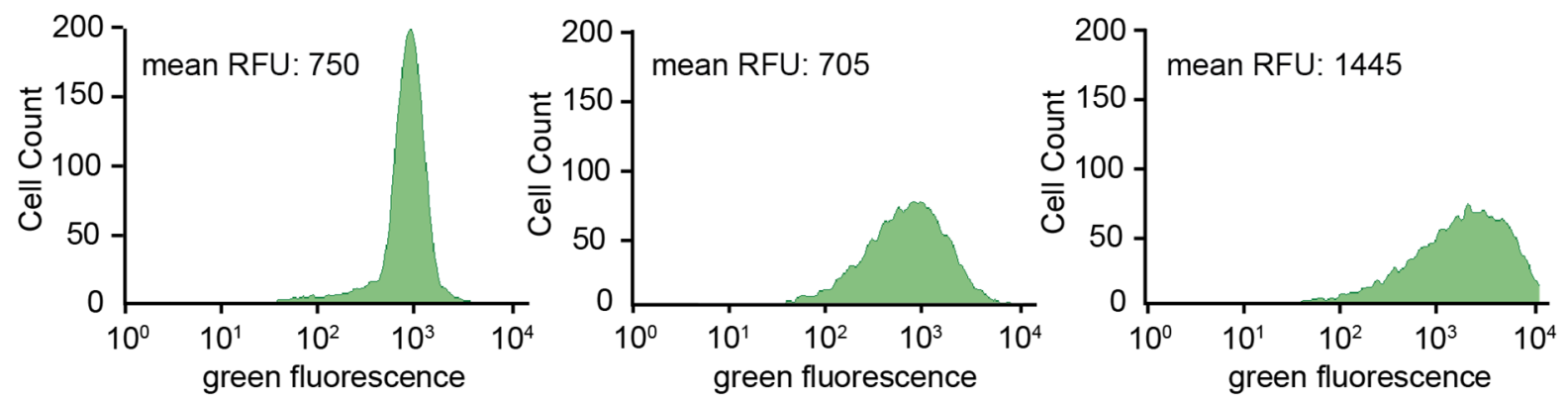

C.

stable HGM HeLa

AAV-HGM-HeLa

AAV-H2GH-HeLa
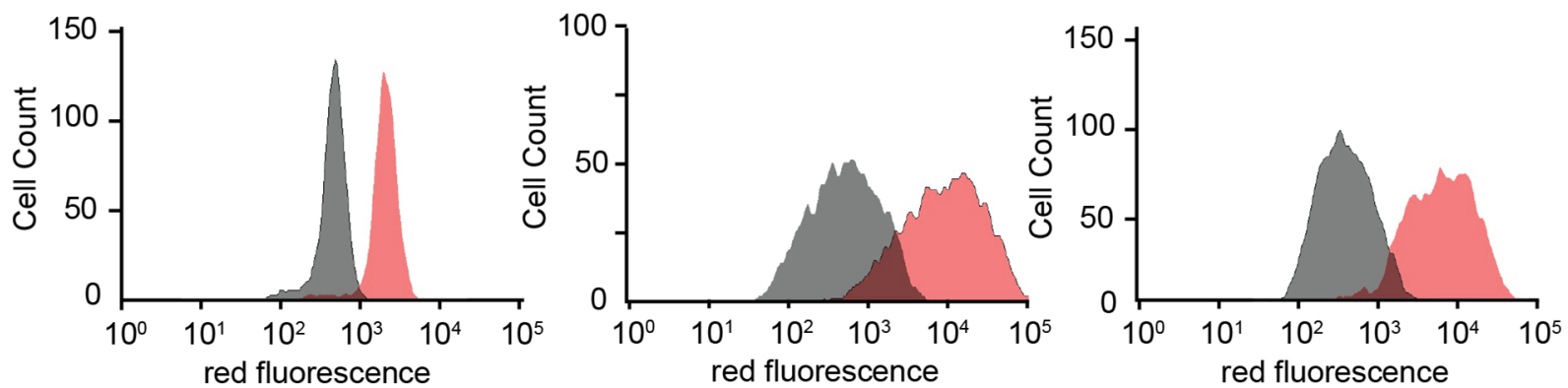

SI Figure 14. Transduction of HeLa cells with novel AAV vectors AAV-HGM and AAV-H2GH. A. Flow cytometry plots showing side scatter versus green fluorescence for a population of stable HGM HeLa cells, AAV-HGM-transduced HeLa cells, and AAV-H2GH-transduced HeLa cells. Gate for green fluorescence was determined based on an untreated population of HeLa cells. Cells expressing substantial levels of GFP were included in a further analysis. B. Flow cytometry histograms of cells gated for live cells and green fluorescence. The stable cells have a relatively homogenous expression of HaloTag-GFP while the AAVtransduced cells have a slightly broader range of expression. C. Flow cytometry histograms of HGM and AAV-transduced HeLa cells treated with chloroalkane-tagged dye (ct-TMR, SI Figure 1) shown in red, compared to background red fluorescence of the cell populations shown in gray. 


\begin{tabular}{lccc}
\hline & $\begin{array}{c}\mathrm{CP}_{50}(\mu \mathrm{M}) \\
\text { Stable HGM HeLa }\end{array}$ & $\begin{array}{c}\mathrm{CP}_{50}(\mu \mathrm{M}) \\
\text { AAV-HGM HeLa }\end{array}$ & $\begin{array}{c}\mathrm{CP}_{50}(\mu \mathrm{M}) \\
\text { AAV-H2GH HeLa }\end{array}$ \\
\hline ct-W & $0.02 \pm 0.002$ & $0.07 \pm 0.01$ & $0.04 \pm 0.01$ \\
\hline ct-Tat & $6.0 \pm 0.6$ & $8.0 \pm 1.7$ & $7.5 \pm 2.1$ \\
\hline ct-R9W & $0.22 \pm 0.02$ & $0.23 \pm 0.01$ & $0.42 \pm 0.05$ \\
\hline
\end{tabular}

stable HGM HeLa

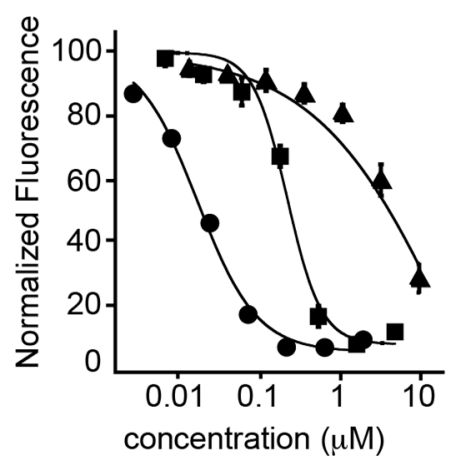

AAV-HGM-HeLa

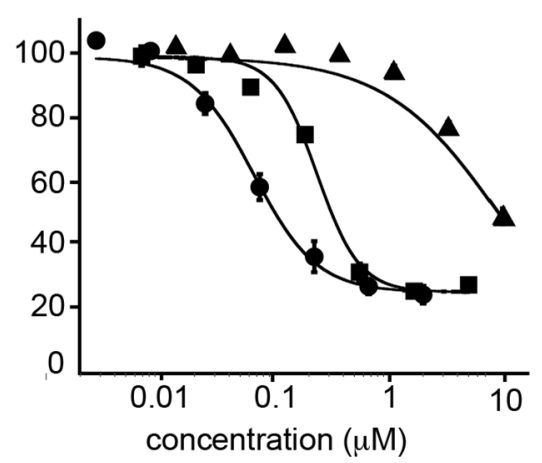

AAV-H2GH-HeLa

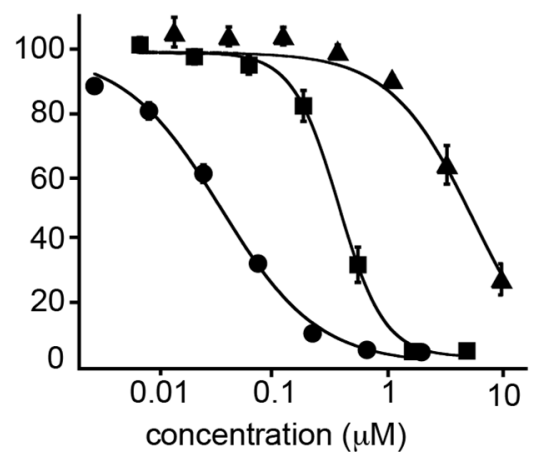

SI Figure 15. Benchmarking CAPA in AAV-transduced HeLa cells using control compounds. Dosedependent CAPA curves and $\mathrm{CP}_{50}$ values for three control compounds in stable HGM cells, HeLa transduced with AAV-HGM, and HeLa transduced with AAV-H2GH. Cells were pulsed with the indicated compound for $4 \mathrm{~h}$ at $37^{\circ} \mathrm{C}$ in serum-free medium, chased with ct-TMR, and analyzed by flow cytometry. CAPA curves and $\mathrm{CP}_{50}$ values are similar for each of these cell lines, indicating that CAPA works reproducibly and accurately in AAV-transduced HeLa cell lines. ct-W is a chloroalkane-labeled tryptophan, ct-Tat is chloroalkane-tagged peptide YGRKKRRQRRR and ct-R9W is chloroalkane-tagged peptide RRRRRRRRRW (SI Figure 1). Each of these ct-compounds are well-established controls for CAPA. ${ }^{1,2}$ Error bars represent the standard error of the mean for three independent replicates. $\mathrm{CP}_{50}$ values are the averages and standard errors calculated from three independent curve fits. 
A.

\begin{tabular}{|ccccc|}
\hline & Name & Modality & Backbone & Sugars \\
\hline- & ct-W & small molecule & n/a & n/a \\
\hline- & A-6 & ASO & PS, stereorandom & $2 \mathrm{MOE}$, all \\
\hline- C-11 & ASO & PMO & PMO \\
\hline E & D-6 & siRNA & PO, 6PS, cholesterol & $2 \mathrm{OMe}, 2 \mathrm{~F}$ alternating \\
\hline- & E-2 & ASO & PS, stereopure & $2 \mathrm{Me}$, gapmer \\
\hline
\end{tabular}

B.

Stable HGM HeLa

no serum

\begin{tabular}{|cc|}
\hline Name & CP $_{50}(\mu \mathrm{M})$ \\
\hline ct-W & $0.008 \pm 0.002$ \\
\hline A-6 & $0.50 \pm 0.11$ \\
\hline C-11 & $0.48 \pm 0.13$ \\
\hline D-6 & $0.12 \pm 0.03$ \\
\hline- E-2 & $0.11 \pm 0.03$ \\
\hline
\end{tabular}

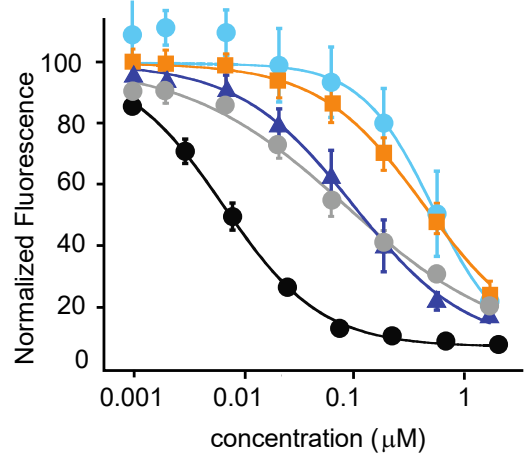

\begin{tabular}{cc|}
\multicolumn{1}{c}{$\begin{array}{c}\text { AAV-H2GH HeLa } \\
\text { no serum }\end{array}$} \\
\hline Name & CP $_{50}(\mu \mathrm{M})$ \\
\hline- ct-W & $0.006 \pm 0.001$ \\
\hline A-6 & $0.27 \pm 0.06$ \\
\hline C-11 & $0.78 \pm 0.30$ \\
\hline D-6 & $0.11 \pm 0.04$ \\
\hline- E-2 & $0.26 \pm 0.12$ \\
\hline
\end{tabular}

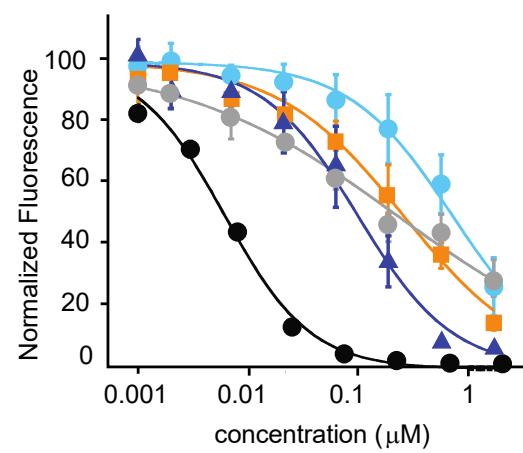

D.

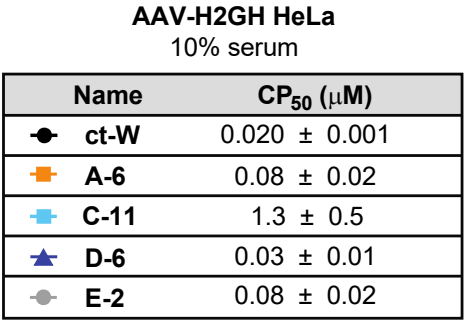

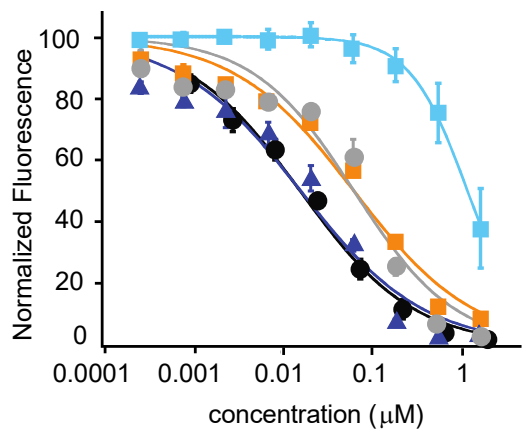

E.

HeLa, cytosol, no serum

$\square$ HeLa, nucleus, no serum

$\square$ HeLa, nucleus, $10 \%$ serum

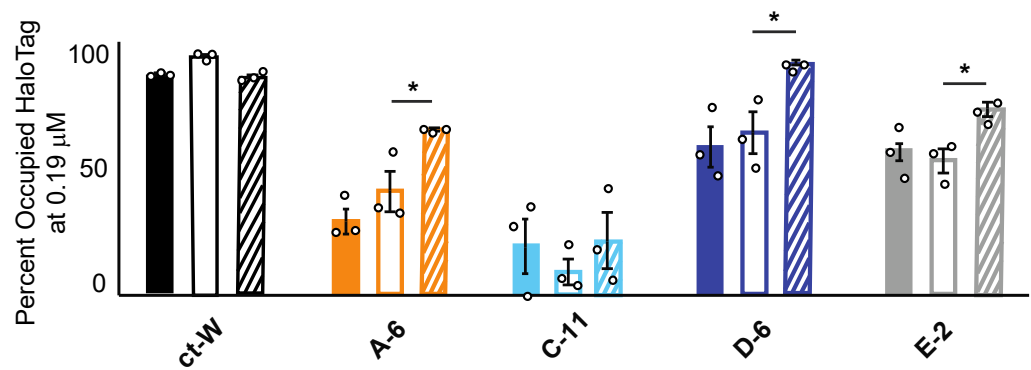

SI Figure 16. Analyzing nuclear penetration and effects of serum for chemically modified RNAs. A. Compounds used in CAPA experiments measuring nuclear penetration. ct-W is a chloroalkane-tagged small molecule, compound A-6 is a PS-2MOE modified ASO, compound D-2 is a PS-2OMe modified gapmer ASO, compound E-5 is a cholesterol-conjugated siRNA, and compound C-11 is a PMO-modified ASO. See SI Table 1 for complete sequences and modifications. B. Full dose-dependent CAPA curves using HGM HeLa cells, which measures the cytosolic localization of compounds. Cells were incubated for $24 \mathrm{~h}$ in serum-free media. C. Full dose-dependent CAPA curves using AAV-H2GH-transduced HeLa cells, which measures the nuclear localization of compounds. Cells were incubated for $24 \mathrm{~h}$ in serum-free media. 
D. Full dose-dependent CAPA curves using AAV-H2GH-transduced HeLa cells in media containing $10 \%$ serum. E. Percent occupied HaloTag at $0.19 \mu \mathrm{M}$ of applied compound for the indicated experimental condition and compartment. HGM cells were used for cytosolic penetration (closed box) and AAV-H2GHtransduced HeLa cells were used for nuclear penetration (open box for serum-free incubation and striped box for incubation in $10 \%$ serum). No significant differences were observed between cytosolic and nuclear penetration for any compound tested ( $p$ values $>0.05$ ). Significant differences in CAPA signal were observed between the serum-free and serum conditions for compounds A-6, E-2, and D-6 ( $p$ value < 0.05). Significance was determined using a two-tailed t-test. Error bars represent the standard error from three independent trials. See SI Figure 18 for full CAPA curves for each condition. All $\mathrm{CP}_{50}$ values are listed in SI Table 8. 


\begin{tabular}{|ccccc|}
\hline & Name & \# Strands & Backbone & Sugars \\
\hline- & ct-W & n/a & n/a & n/a \\
\hline- & A-6 & 1 & PS, stereorandom & 2MOE, all \\
\hline- & C-11 & 1 & PMO & PMO \\
\hline+ & D-6 & 2 & PO, 6PS, cholesterol & 2OMe, 2F alternating \\
\hline- & E-2 & 1 & PS, stereopure & 2OMe, gapmer \\
\hline
\end{tabular}

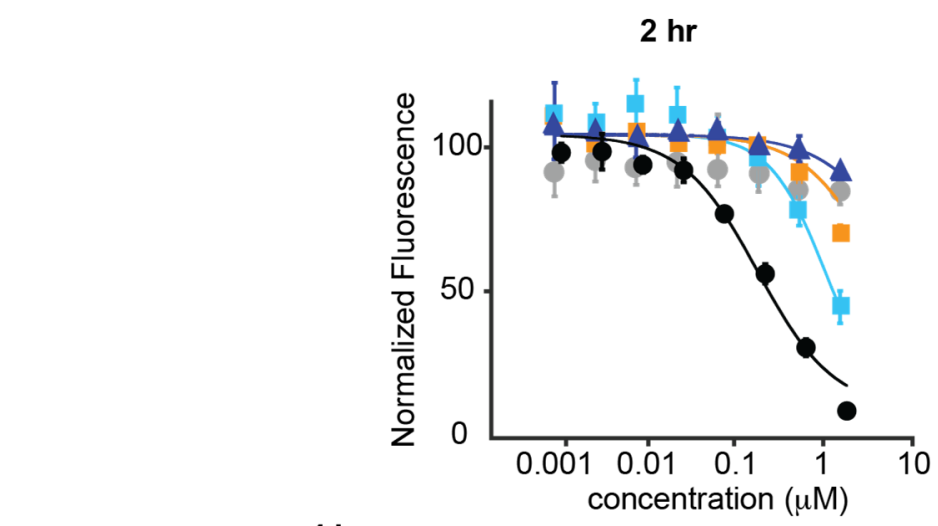

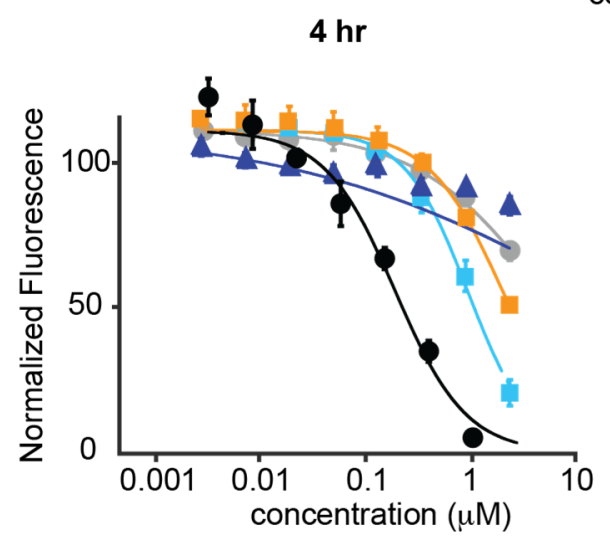

$24 \mathrm{hr}$

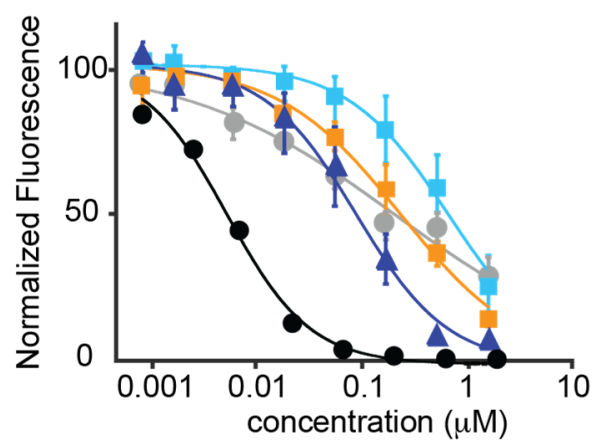

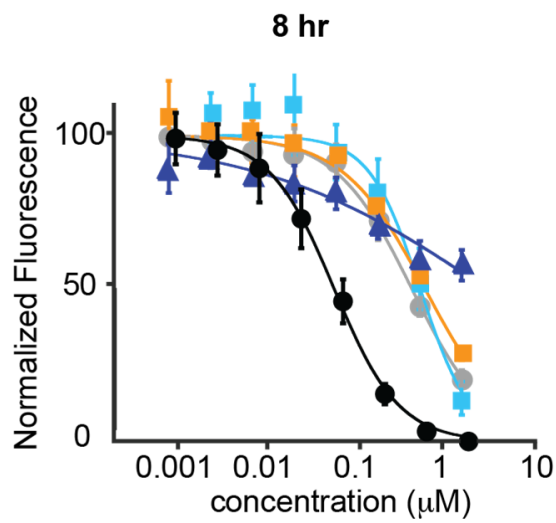

$48 \mathrm{hr}$

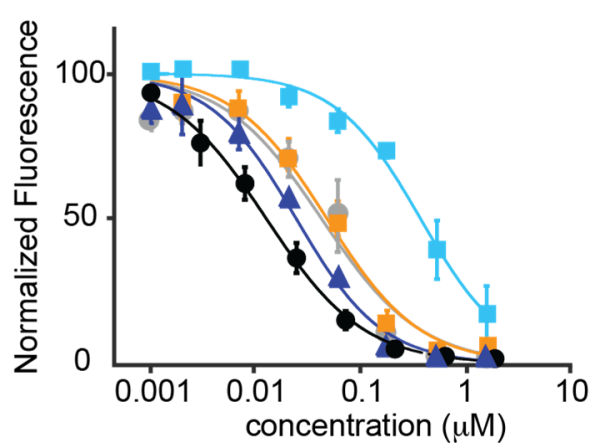

SI Figure 17. Time course of nuclear localization of modified RNAs, grouped by incubation time. Full dose-dependent CAPA curves for each compound with AAV-H2GH-transduced cells. These are the same data shown in SI Figure 18 and main text Figure 4 but grouped by incubation time. Error bars represent the standard error from three independent trials. 


\begin{tabular}{|lccc|}
\hline Name & \# Strands & Backbone & Sugars \\
\hline ct-W & n/a & n/a & n/a \\
\hline A-6 & 1 & PS, stereorandom & 2MOE, all \\
\hline C-11 & 1 & PMO & PMO \\
\hline D-6 & 2 & PO, 6PS, cholesterol & 2OMe, 2F alternating \\
\hline E-2 & 1 & PS, stereopure & 2OMe, gapmer \\
\hline
\end{tabular}

\section{Incubation Time (hr)}

$\begin{array}{ll}-2 \mathrm{hr} & -24 \mathrm{hr} \\ -4 \mathrm{hr} & -48 \mathrm{hr} \\ -8 \mathrm{hr} & \end{array}$

A-6

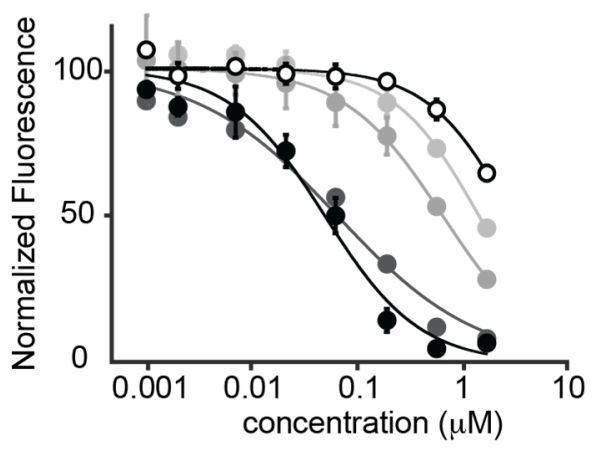

D-6

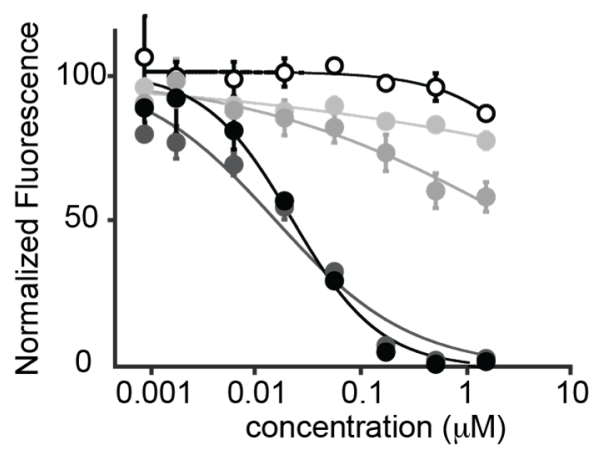

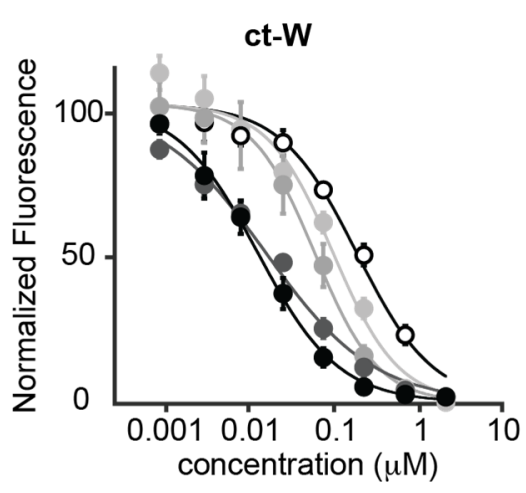

E-2
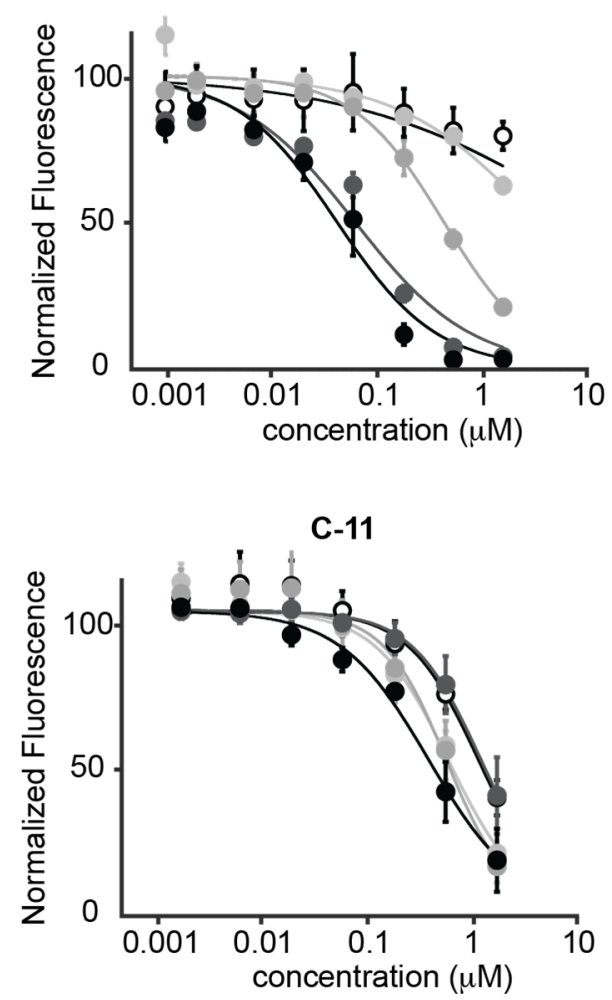

SI Figure 18. Time course of nuclear localization of modified RNAs, grouped by compound. Full dose-dependent CAPA curves for each compound with AAV-H2GH-transduced cells. These are the same data shown in SI Figure 17 and main text Figure 4 but grouped by compound. Error bars represent the standard error from three independent trials. 
A.

$\operatorname{ct}(\mathrm{PO})$ Linker

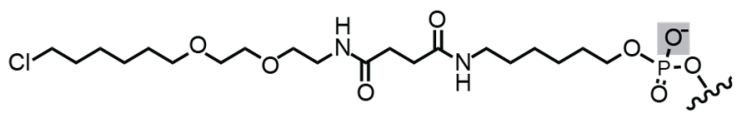

ct(PS) Linker

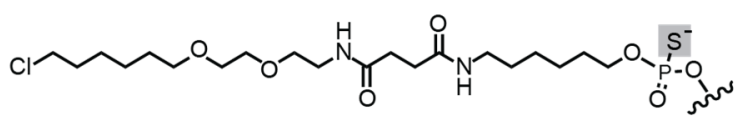

B.

\begin{tabular}{|c|c|c|c|c|c|}
\hline Name & Linker & Backbone & Sugars & $\begin{array}{c}\mathrm{CP}_{50}(u \mathrm{M}) \\
\text { Serum }\end{array}$ & $\begin{array}{l}\mathrm{CP}_{50}(\mu \mathrm{M}) \\
\text { No Serum }\end{array}$ \\
\hline$\rightarrow A-6$ & $\operatorname{ct}(\mathrm{PO})-$ & stereorandom & $2 \mathrm{MOE}$, all & $0.08 \pm 0.02$ & $0.50 \pm 0.11$ \\
\hline $\pm A-8$ & ct(PS)- & stereorandom & 2MOE, all & $0.35 \pm 0.03$ & $2.64 \pm 0.91$ \\
\hline$-B-5$ & $\operatorname{ct}(\mathrm{PO})-$ & stereorandom & 2OMe, gapmer & $0.08 \pm 0.02$ & $0.13 \pm 0.08$ \\
\hline$\pm \mathrm{B}-6$ & $\operatorname{ct}(\mathrm{PS})-$ & stereorandom & 2OMe, gapmer & $7.6 \pm 6.1$ & $>20$ \\
\hline$\rightarrow C-1$ & $\operatorname{ct}(\mathrm{PO})-$ & stereorandom & $2 \mathrm{OMe}$, all & $0.07 \pm 0.01$ & $0.09 \pm 0.003$ \\
\hline$\pm \quad C-13$ & $\operatorname{ct}(P S)-$ & stereorandom & 2OMe, all & $>20$ & $>20$ \\
\hline$-C-12$ & $\operatorname{ct}(\mathrm{PO})-$ & stereopure & $2 \mathrm{~F}$, all & $0.06 \pm 0.01$ & $0.02 \pm 0.002$ \\
\hline$\pm \quad C-14$ & $\operatorname{ct}(P S)-$ & stereopure & $2 \mathrm{~F}$, all & $7.2 \pm 3.5$ & $>20$ \\
\hline$-E-1$ & ct(PO)- & stereorandom & 2OMe, gapmer & $0.08 \pm 0.01$ & $0.19 \pm 0.07$ \\
\hline$\pm \mathrm{E}-3$ & $\operatorname{ct}(\mathrm{PS})-$ & stereorandom & 2OMe, gapmer & $10.5 \pm 8.0$ & $>20$ \\
\hline$-E-2$ & ct(PO)- & stereopure & 2OMe, gapmer & $0.12 \pm 0.06$ & $0.38 \pm 0.27$ \\
\hline$-E-4$ & ct(PS)- & stereopure & 2OMe, gapmer & $3.7 \pm 1.5$ & $>20$ \\
\hline
\end{tabular}

C. $\operatorname{ct}(P O)$, no serum $\quad$ o. $\operatorname{ct}(P O), 10 \%$ serum $\operatorname{ct}(P S)$, no serum

$\triangle \operatorname{ct}(P S), 10 \%$ serum
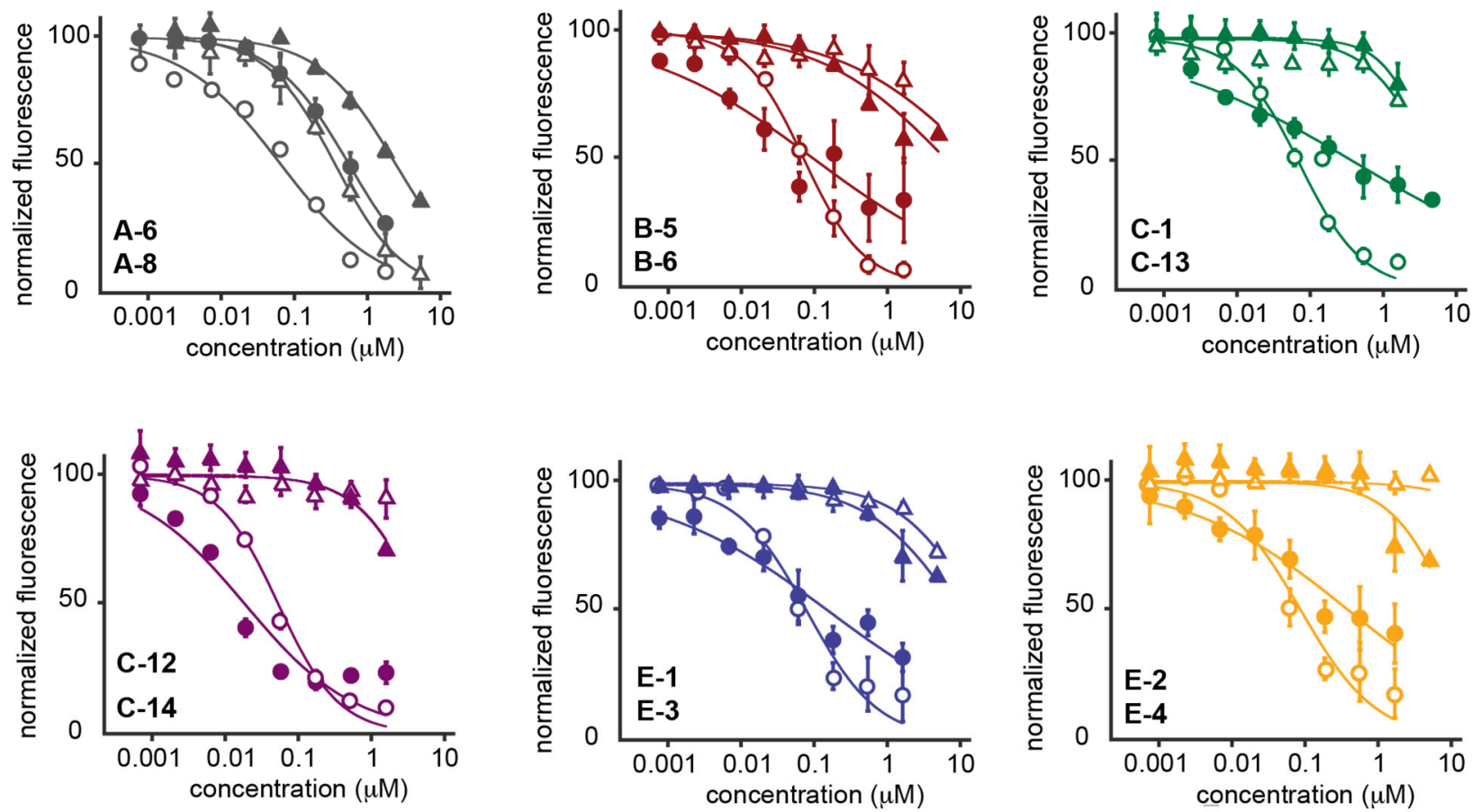

SI Figure 19. Impact of chloroalkane linker on cytosolic localization. A. Chemical structure of the $\mathrm{ct}(\mathrm{PO})$ and $\mathrm{ct}(\mathrm{PS})$ linkers. B. Table of compounds and $\mathrm{CP}_{50}$ values. C. Full CAPA curves for each compound 
after a $24 \mathrm{~h}$ incubation with HGM cells. Closed circles represent compounds with the ct(PO) linker in serumfree media, and open circles represent compounds with the ct(PO) linker in media containing $10 \%$ serum. Closed triangles represent compounds with the $\mathrm{ct}(\mathrm{PS})$ linker in serum-free media, and open triangles represent compounds with the ct(PS) linker in media containing $10 \%$ serum. Sequences and chemical modifications of these compounds are shown in SI Table 1. Error bars represent the standard error of the mean of three independent experiments. $\mathrm{CP}_{50}$ values are averages and standard errors calculated from three independent curve fits. $\mathrm{CP}_{50}$ values are also listed in SI Tables 12 and 13. 
A.

Luciferase Splice Correction:

facilitated uptake, $24 \mathrm{hr}$

\begin{tabular}{|c|c|c|c|c|}
\hline & Name & ct-linker & Sugar & Fold $\mathrm{RLU}$ at $30 \mathrm{nM}$ \\
\hline- & C-1 & $\operatorname{ct}(\mathrm{PO})$ & $2 \mathrm{OMe}$ & $27.6 \pm 4.1$ \\
\hline \pm & C-12 & $\operatorname{ct}(\mathrm{PO})$ & $2 \mathrm{~F}$ & $42.4 \pm 10.0$ \\
\hline- & C-13 & $\operatorname{ct}(P S)$ & $20 \mathrm{Me}$ & $32.4 \pm 0.7$ \\
\hline \pm & C-14 & $\operatorname{ct}(P S)$ & $2 \mathrm{~F}$ & $35.4 \pm 12.2$ \\
\hline
\end{tabular}

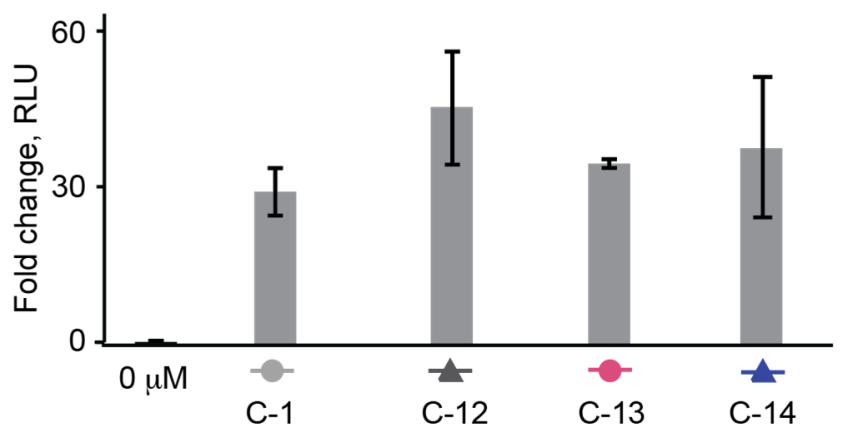

B.

Luciferase Splice Correction: facilitated uptake, $48 \mathrm{hr}$

\begin{tabular}{|ccccc|}
\hline & Name & ct-linker & Sugar & Fold RLU at 30 nM \\
\hline- & C-1 & $\operatorname{ct}(\mathrm{PO})$ & $2 \mathrm{OMe}$ & $7.8 \pm 1.1$ \\
\hline \pm & C-12 & $\operatorname{ct}(\mathrm{PO})$ & $2 \mathrm{~F}$ & $11.6 \pm 4.1$ \\
\hline- & C-13 & $\operatorname{ct}(\mathrm{PS})$ & $2 \mathrm{OMe}$ & $9.7 \pm 1.4$ \\
\hline \pm & C-14 & $\operatorname{ct}(\mathrm{PS})$ & $2 \mathrm{~F}$ & $10.0 \pm 3.7$ \\
\hline
\end{tabular}

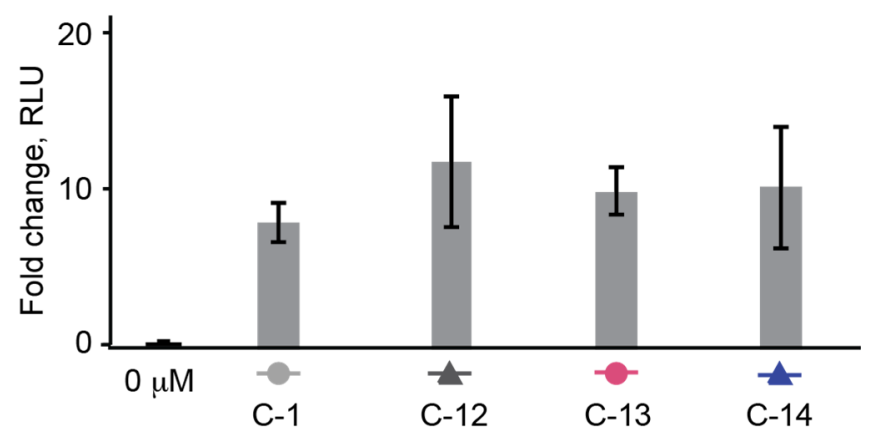

SI Figure 20. Splice-correction activity of selected ASOs after transfection using lipofectamine. Luciferase splice-correction activity calculated as fold change RLU at $30 \mathrm{nM}$ of all oligonucleotides. Cells were incubated with $30 \mathrm{nM}$ ct-ASO complexed with lipofectamine for $4 \mathrm{~h}$ and then media was replaced. The cells were then incubated for a total of A. 24 h or B. 48 h before quantitating luciferase expression. Error bars represent the standard error from three independent trials. 
Luciferase Splice Correction:

gymnotic uptake, $48 \mathrm{hr}, 10 \%$ serum

\begin{tabular}{|ccccc|}
\hline & Name & ct-linker & Sugar & Fold RLU at $5 \mu \mathrm{M}$ \\
\hline- & $\mathrm{C}-1$ & $\mathrm{ct}(\mathrm{PO})$ & $2 \mathrm{OMe}$ & $4.2 \pm 1.1$ \\
\hline \pm & $\mathrm{C}-12$ & $\mathrm{ct}(\mathrm{PO})$ & $2 \mathrm{~F}$ & $5.0 \pm 1.0$ \\
\hline- & $\mathrm{C}-13$ & $\mathrm{ct}(\mathrm{PS})$ & $2 \mathrm{OMe}$ & $2.2 \pm 0.5$ \\
\hline \pm & $\mathrm{C}-14$ & $\mathrm{ct}(\mathrm{PS})$ & $2 \mathrm{~F}$ & $4.1 \pm 0.9$ \\
\hline
\end{tabular}

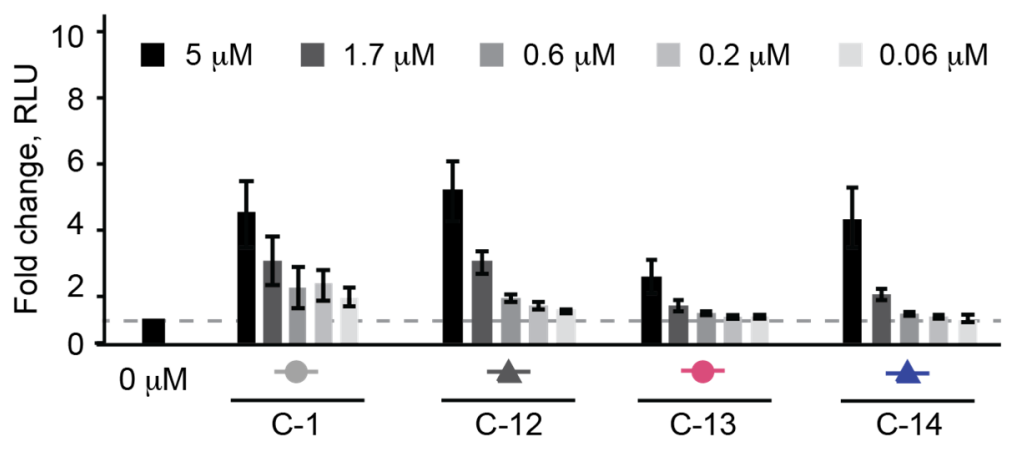

SI Figure 21. Splice-correcting activity of selected ASOs after gymnotic uptake. Cells were incubated with the indicated concentration of ct-ASO for $48 \mathrm{~h}$ in media containing $10 \%$ serum. Error bars represent the standard error from three independent trials. 
A.

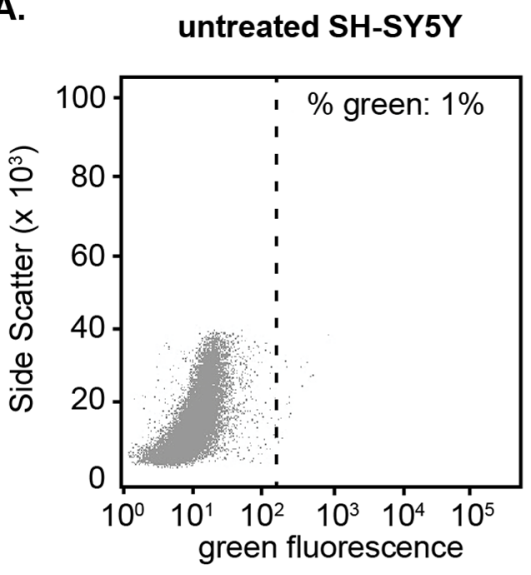

B.

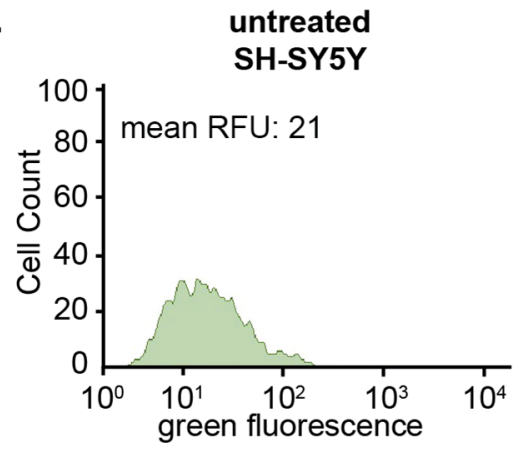

C.

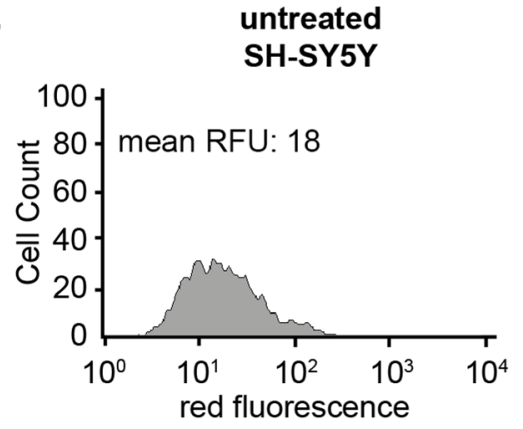

AAV-H2GH SH-SY5Y

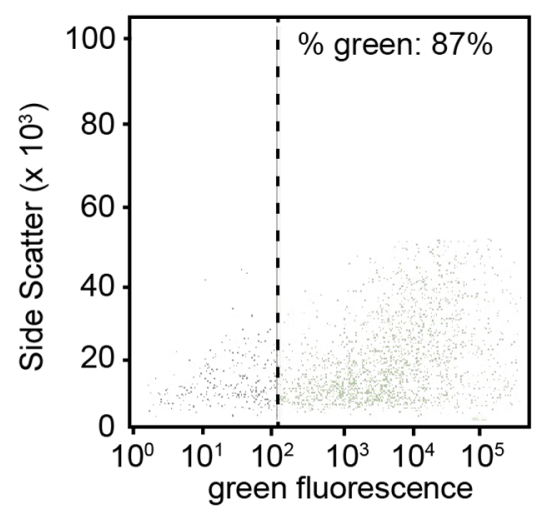

AAV-H2GH

SH-SY5Y
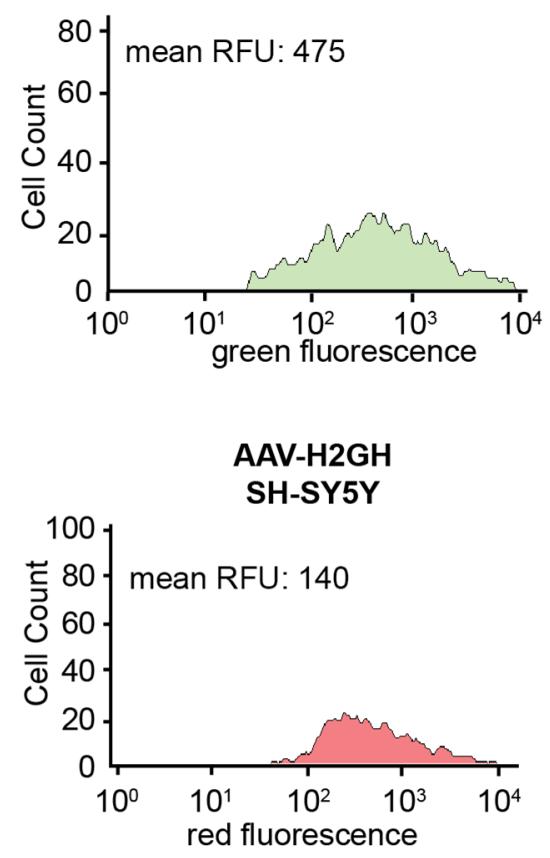

SI Figure 22. Transduction of SH-SY5Y cells with novel AAV vector AAV-H2GH. A. Flow cytometry plots showing side scatter versus green fluorescence of a population of untreated SH-SY5Y cells and AAV$\mathrm{H} 2 \mathrm{GH}$-transduced SH-SY5Y cells. Cells expressing substantial levels of GFP were included in further analysis. B. Flow cytometry histograms of cells gated for live cells and green fluorescence. C. Flow cytometry histograms of untreated and AAV-H2GH-transduced SH-SY5Y cells treated with ct-TMR. AAV$\mathrm{H} 2 \mathrm{GH}$-transduced $\mathrm{SH}-\mathrm{SY} 5 \mathrm{Y}$ cells have a roughly 10 -fold increase in red fluorescence, indicating robust expression of HaloTag. 


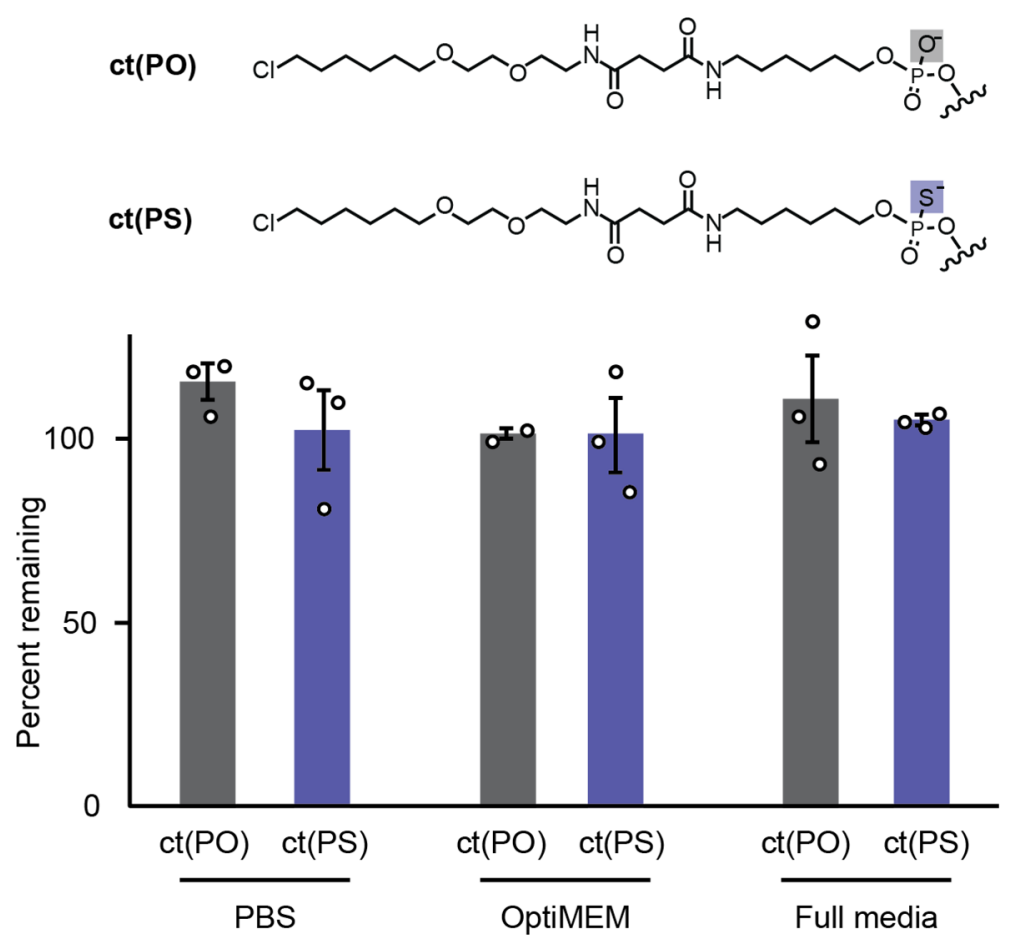

SI Figure 23. Chloroalkane-tagged ASOs are stable in serum-containing media. ASOs tagged with a chloroalkane group via either a 5' phosphodiester or 5' phosphorothioate were incubated in either PBS, OptiMEM, or full media (DMEM plus 10\% serum). Analytical HPLC was used to quantitate percentages of intact ct-ASOs remaining after $24 \mathrm{~h}$ incubation. ct-ASOs have the base sequence of nusinersen (Sequence A: UCACUUUCAUAAUGCUGG). Error bars represent the standard error of the mean from three independent replicates. 
A.

Malat1 knockdown:

gymnotic uptake, $24 \mathrm{hr}$, full media

\begin{tabular}{|c|c|c|c|c|}
\hline & Name & ct-linker & $\begin{array}{c}\text { Stereo- } \\
\text { pure? }\end{array}$ & $I C_{50}(\mu M)$ \\
\hline- & E-1 & $\operatorname{ct}(\mathrm{PO})$ & - & $>100$ \\
\hline \pm & E-2 & $\operatorname{ct}(\mathrm{PO})$ & $\sqrt{ }$ & $7.6 \pm 3.4$ \\
\hline 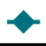 & E-3 & $\mathrm{n} / \mathrm{a}$ & - & $14.8 \pm 4.6$ \\
\hline$\checkmark$ & $E-4$ & $\mathrm{n} / \mathrm{a}$ & $\checkmark$ & $4.3 \pm 0.8$ \\
\hline- & E-5 & $\operatorname{ct}(P S)$ & - & $8.4 \pm 1.7$ \\
\hline \pm & E-6 & $\operatorname{ct}(\mathrm{PS})$ & $\sqrt{ }$ & $7.2 \pm 2.3$ \\
\hline
\end{tabular}

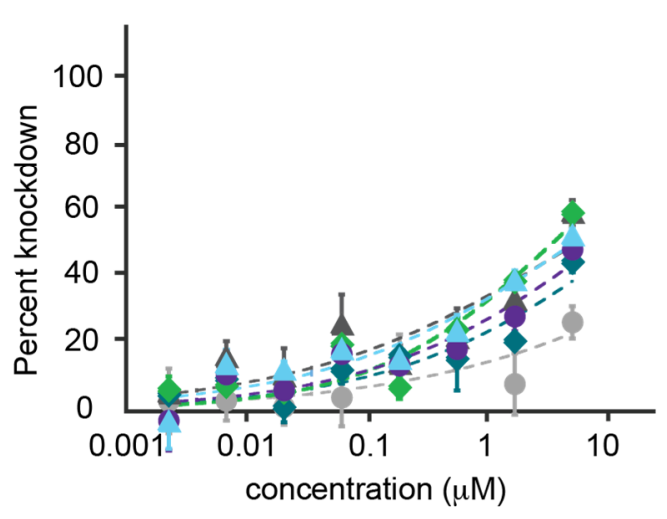

B.

CAPA: AAV-H2GH-SH-SY5Y

gymnotic uptake, $24 \mathrm{hr}$, full media

\begin{tabular}{|ccccc|}
\hline Name & ct-linker & $\begin{array}{c}\text { Stereo- } \\
\text { pure? }\end{array}$ & CP $_{50}(\mu \mathrm{M})$ \\
\hline- & E-1 & $\operatorname{ct}(\mathrm{PO})$ & - & $0.06 \pm 0.003$ \\
\hline \pm & $\mathrm{E}-2$ & $\operatorname{ct}(\mathrm{PO})$ & $\checkmark$ & $0.14 \pm 0.07$ \\
\hline- & $\mathrm{E}-5$ & $\operatorname{ct}(\mathrm{PS})$ & - & $0.19 \pm 0.06$ \\
\hline \pm & $\mathrm{E}-6$ & $\mathrm{ct}(\mathrm{PS})$ & $\checkmark$ & $0.30 \pm 0.09$ \\
\hline- & ct-W & $\mathrm{n} / \mathrm{a}$ & $\mathrm{n} / \mathrm{a}$ & $0.26 \pm 0.19$ \\
\hline
\end{tabular}

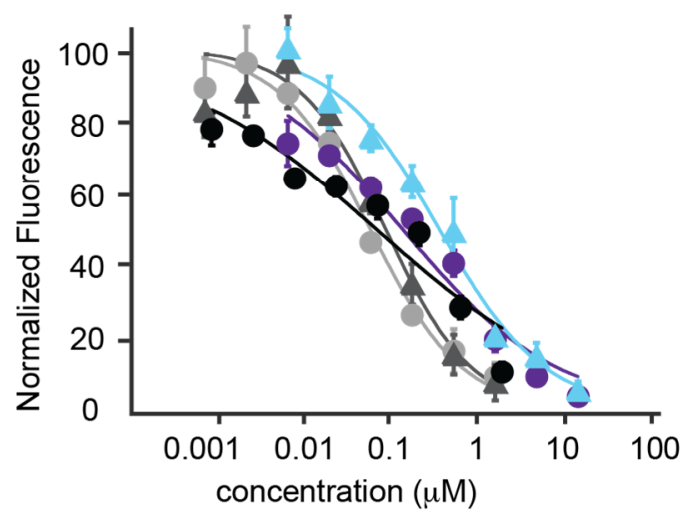

SI Figure 24. CAPA and knockdown activity in SH-SY5Y cells. A. MALAT1 knockdown activity in SHSY5Y cells. Cells were incubated with the indicated concentration of compound for $24 \mathrm{~h}$ in full media with $10 \%$ serum. B. Dose-dependent CAPA curves and $\mathrm{CP}_{50}$ values for MALAT1 knockdown. AAV-H2GH SHSY5Y cells were incubated with the indicated concentration of compound for $24 \mathrm{~h}$ in media containing $10 \%$ serum. Sequences and chemical modifications of these compounds are shown in SI Table 1. CP 50 values are also listed in SI Table 11. 

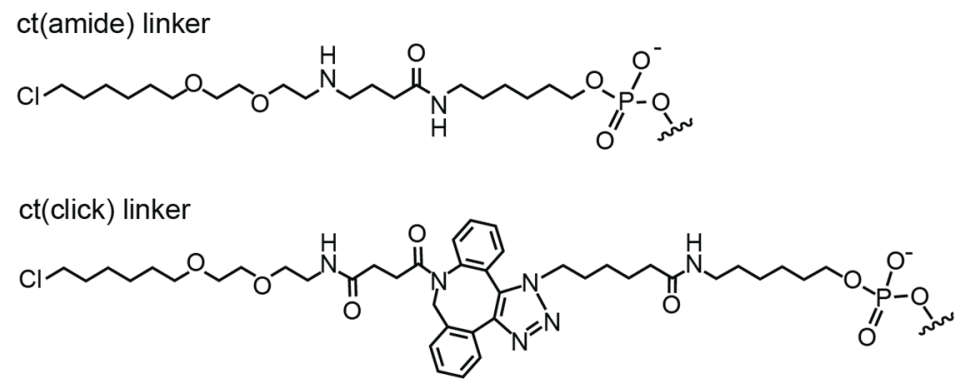

\begin{tabular}{|ccccc|}
\hline & Name & Modifications & Linker & CP $_{50}(\mu \mathrm{M})$ \\
\hline- & A-6 & PS-2MOE & ct(amide) & $3.98 \pm 0.21$ \\
\hline \pm & A-7 & PS-2MOE & ct(click) & $0.76 \pm 0.31$ \\
\hline- & B-4 & PS-2OMe & ct(amide) & $1.51 \pm 0.23$ \\
\hline \pm & B-7 & PS-2OMe & ct(click) & $0.49 \pm 0.18$ \\
\hline- & C-11 & PMO & ct(amide) & $1.37 \pm 0.13$ \\
\hline \pm & C-15 & PMO & ct(click) & $1.27 \pm 0.33$ \\
\hline
\end{tabular}
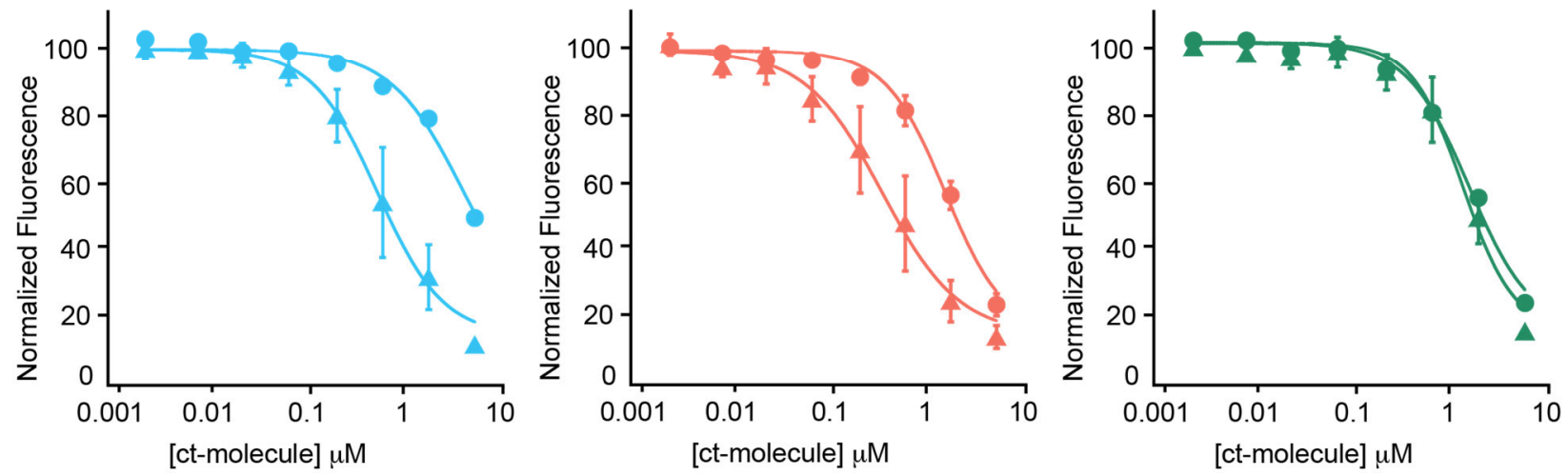

SI Figure 25. Comparison of cytosolic localization for ASOs with amide and click linkers. The ct(PO)amide linker results from the conjugation of a chloroalkane-carboxylic acid with an oligonucleotide prepared with a $5^{\prime}$ amine through an amide coupling (see Methods). The ct(PO)-click linker results from the conjugation of a chloroalkane-dibenzocyclooctyne with an oligonucleotide prepared with a 5 ' azide through a copper-free click reaction coupling (see Methods). Phosphorothioate backbones are denoted PS, phosphorodiamidate morpholino modifications are denoted PMO, sugars modified with 2'-O-methyl modifications are denoted $2 \mathrm{OMe}$, and sugars modified with 2'-O-methoxyethyl modifications are denoted $2 \mathrm{MOE}$. Cells were incubated with the indicated concentration of ct-ASO for $4 \mathrm{~h}$ in serum-free media. Compound A-7 is approximately 5 -fold more cell-penetrant under these experimental conditions than compound A-6 ( $p$ value <0.05), and compound B-7 is approximately 3-fold more cell-penetrant than compound B-4 ( $p$ value <0.05), suggesting that the click linker promotes a higher degree of cytosolic localization than the amide linker. No significant difference was observed for the different linkers for the PMO compounds $\mathbf{C}-11$ and $\mathbf{C}-15$. Significance was determined using a two-tailed t-test. $\mathbf{C P}_{50}$ values are averages and standard errors calculated from three independent curve fits and are also listed in SI Table 14. 
SI Table 2. $\mathrm{CP}_{50}$ values from experiments with drug-like ASOs. HGM HeLa cells were incubated with the indicated compounds for $4 \mathrm{~h}$ in serum-free media. Error represents the standard error from three independent replicates. Values correspond to data in main text Figure 1 and SI Figure 2.

\begin{tabular}{|c|c|}
\hline ct-compound & $\mathrm{CP}_{50}(\mu \mathrm{M})$ \\
\hline ct-W & $0.05 \pm 0.01$ \\
\hline A-1 & $10.1 \pm 3.5$ \\
\hline A-3 & $4.0 \pm 0.8$ \\
\hline A-5 & $3.2 \pm 1.3$ \\
\hline A-2 & $11.1 \pm 4.6$ \\
\hline A-4 & $1.3 \pm 0.1$ \\
\hline A-6 & $4.2 \pm 0.6$ \\
\hline B-1 & $4.2 \pm 1.1$ \\
\hline B-3 & $11.9 \pm 5.3$ \\
\hline B-2 & $2.6 \pm 0.4$ \\
\hline B-4 & $0.6 \pm 0.04$ \\
\hline
\end{tabular}

SI Table 3. $\mathrm{CP}_{50}$ values from CAPA experiments exploring the effects of patterning. HGM HeLa cells were incubated with the indicated compounds for $4 \mathrm{~h}$ in serum-free media. Error represents the standard error from three independent replicates. Values correspond to data in main text Figure 2.

\begin{tabular}{|c|c|}
\hline ct-compound & $\mathrm{CP}_{50}(\mu \mathrm{M})$ \\
\hline ct-W & $0.05 \pm 0.01$ \\
\hline C-1 & $0.59 \pm 0.14$ \\
\hline C-2 & $3.51 \pm 0.47$ \\
\hline C-3 & $0.46 \pm 0.12$ \\
\hline C-4 & $7.14 \pm 0.48$ \\
\hline C-5 & $5.06 \pm 0.54$ \\
\hline C-6 & $0.72 \pm 0.13$ \\
\hline C-7 & $1.14 \pm 0.27$ \\
\hline C-8 & $>15$ \\
\hline C-9 & $0.34 \pm 0.04$ \\
\hline C-10 & $>15$ \\
\hline
\end{tabular}

SI Table 4. $\mathrm{CP}_{50}$ values from CAPA experiments with siRNAs. HGM HeLa cells were incubated with the indicated compounds for $48 \mathrm{~h}$ in serum-free media. Error represents the standard error from three independent replicates. Values correspond to data in main text Figure 3B.

\begin{tabular}{|c|c|}
\hline ct-compound & $\mathrm{CP}_{50}(\mu \mathrm{M})$ \\
\hline ct-W & $0.012 \pm 0.004$ \\
\hline D-2 & $0.11 \pm 0.01$ \\
\hline D-3 & $0.04 \pm 0.01$ \\
\hline D-4 & $>15$ \\
\hline D-6 & $0.25 \pm 0.05$ \\
\hline D-7 & $0.03 \pm 0.01$ \\
\hline D-9 & $0.04 \pm 0.01$ \\
\hline D-10 & $0.03 \pm 0.01$ \\
\hline D-11 & $0.04 \pm 0.01$ \\
\hline
\end{tabular}


SI Table 5. $\mathrm{CP}_{50}$ values from CAPA experiments in HeLa cells 24 hours after transduction. AAV$\mathrm{H} 2 \mathrm{GH}$ HeLa cells were incubated with the indicated compounds for $4 \mathrm{~h}$ in serum-free media. Error represents the standard error from three independent replicates. Values correspond to data in SI Figure 14.

\begin{tabular}{|c|c|c|c|}
\hline ct-compound code & $\begin{array}{c}\text { Stable HGM } \\
C P_{50}(\mu \mathrm{M})\end{array}$ & $\begin{array}{c}A A V-H G M \\
C P_{50}(\mu M)\end{array}$ & $\begin{array}{c}A A V-H 2 G H \\
C P_{50}(\mu M)\end{array}$ \\
\hline ct-W & $0.016 \pm 0.002$ & $0.07 \pm 0.01$ & $0.04 \pm 0.01$ \\
\hline ct-Tat & $6.0 \pm 0.6$ & $8.0 \pm 1.7$ & $7.5 \pm 2.1$ \\
\hline ct-R9W & $0.22 \pm 0.02$ & $0.23 \pm 0.01$ & $0.42 \pm 0.05$ \\
\hline
\end{tabular}

SI Table 6. $\mathrm{CP}_{50}$ values from CAPA experiments in HeLa cells 48 hours after transduction. AAV$\mathrm{H} 2 \mathrm{GH}$ HeLa cells were with the indicated compounds incubated for $4 \mathrm{~h}$ in serum-free media. Error represents the standard error from three independent replicates.

\begin{tabular}{|c|c|c|c|}
\hline ct-compound code & $\begin{array}{c}\text { Stable } H G M \\
C P_{50}(\mu M)\end{array}$ & $\begin{array}{c}A A V-H G M \\
C P_{50}(\mu M)\end{array}$ & $\begin{array}{c}A A V-H 2 G H \\
C P_{50}(\mu M)\end{array}$ \\
\hline ct-W & n/a & $0.07 \pm 0.01$ & n/a \\
\hline ct-Tat & n/a & $8.3 \pm 1.9$ & n/a \\
\hline ct-R9W & n/a & $0.10 \pm 0.02$ & n/a \\
\hline
\end{tabular}

SI Table 7. $\mathrm{CP}_{50}$ values from CAPA experiments in HeLa cells $\mathbf{7 2}$ hours after transduction. AAV$\mathrm{H} 2 \mathrm{GH}$ HeLa cells were incubated with the indicated compounds for $4 \mathrm{~h}$ in serum-free media. Error represents the standard error from three independent replicates.

\begin{tabular}{|c|c|c|c|}
\hline ct-compound code & $\begin{array}{c}\text { Stable } H G M \\
C P_{50}(\mu M)\end{array}$ & $\begin{array}{c}A A V-H G M \\
C P_{50}(\mu M)\end{array}$ & $\begin{array}{c}A A V-H 2 G H \\
C P_{50}(\mu M)\end{array}$ \\
\hline ct-W & n/a & n/a & $0.04 \pm 0.01$ \\
\hline ct-Tat & n/a & n/a & $4.52 \pm 0.63$ \\
\hline ct-R9W & $\mathrm{n} / \mathrm{a}$ & $\mathrm{n} / \mathrm{a}$ & $0.13 \pm 0.02$ \\
\hline
\end{tabular}

SI Table 8. $\mathrm{CP}_{50}$ values from CAPA experiments in stable and transduced cell lines. The indicated cell line was incubated with the indicated compounds for $24 \mathrm{~h}$ under the indicated serum conditions. Error represents the standard error from three independent replicates. Values correspond to data in main text Figures 4 and 6 .

\begin{tabular}{|c|c|c|c|c|}
\hline ct-compound & $\begin{array}{c}C P_{50}(\mu M) \\
H G M \\
H e L a \\
\text { No serum }\end{array}$ & $\begin{array}{c}C P_{50}(\mu M) \\
A A V-H 2 G H \\
H e L a \\
\text { No serum }\end{array}$ & $\begin{array}{c}C P_{50}(\mu M) \\
A A V-H 2 G H \\
H e L a \\
10 \% \text { serum }\end{array}$ & $\begin{array}{c}C P_{50}(\mu M) \\
A A V-H 2 G H \\
S H-S Y 5 Y \\
10 \% \text { serum }\end{array}$ \\
\hline ct-W & $0.008 \pm 0.002$ & $0.006 \pm 0.001$ & $0.020 \pm 0.001$ & $0.15 \pm 0.08$ \\
\hline$C-11$ & $0.48 \pm 0.13$ & $0.78 \pm 0.3$ & $1.34 \pm 0.47$ & $0.11 \pm 0.04$ \\
\hline A-6 & $0.50 \pm 0.11$ & $0.27 \pm 0.06$ & $0.08 \pm 0.02$ & $0.04 \pm 0.02$ \\
\hline E-2 & $0.11 \pm 0.03$ & $0.26 \pm 0.12$ & $0.08 \pm 0.02$ & $0.04 \pm 0.01$ \\
\hline D-6 & $0.12 \pm 0.03$ & $0.11 \pm 0.04$ & $0.025 \pm 0.007$ & $0.11 \pm 0.05$ \\
\hline
\end{tabular}


SI Table 9. $\mathrm{CP}_{50}$ values from time course CAPA experiments. AAV-H2GH HeLa cells were incubated with the indicated compounds in media containing $10 \%$ serum. Error represents the standard error from three independent replicates. Values correspond to data in main text Figure 4.

\begin{tabular}{|c|c|c|c|c|c|}
\hline ct-compound & $\begin{array}{c}2 h \\
C P_{50}(\mu \mathrm{M})\end{array}$ & $\begin{array}{c}4 h \\
C P_{50}(\mu \mathrm{M})\end{array}$ & $\begin{array}{c}8 \mathrm{C} \\
\mathrm{CP}\end{array}$ & $\begin{array}{c}24 \mathrm{~h}(\mu \mathrm{M}) \\
C P_{50}(\mu \mathrm{M})\end{array}$ & $\begin{array}{c}48 \mathrm{~h} \\
\mathrm{CP}\end{array}$ \\
\hline ct-W & $0.18 \pm 0.01$ & $0.09 \pm 0.002$ & $0.06 \pm 0.02$ & $0.02 \pm 0.001$ & $0.01 \pm 0.003$ \\
\hline C-11 & $1.14 \pm 0.23$ & $0.60 \pm 0.11$ & $0.56 \pm 0.19$ & $1.34 \pm 0.47$ & $0.47 \pm 0.15$ \\
\hline A-6 & $4.55 \pm 0.97$ & $1.42 \pm 0.03$ & $0.64 \pm 0.09$ & $0.08 \pm 0.02$ & $0.05 \pm 0.02$ \\
\hline E-2 & $31.6 \pm 25.3$ & $12.6 \pm 9.9$ & $0.44 \pm 0.08$ & $0.08 \pm 0.02$ & $0.05 \pm 0.02$ \\
\hline D-6 & $9.17 \pm 3.3$ & $182 \pm 107$ & $3.27 \pm 1.3$ & $0.02 \pm 0.01$ & $0.02 \pm 0.003$ \\
\hline
\end{tabular}

SI Table 10. CAPA-informed conditions for sufficient nuclear penetration. Minimum concentrations and timepoints required of each compound to achieve nuclear penetration sufficient to obtain at least $80 \%$ HaloTag occupancy. Values are derived from the data shown in main text Figure 4.

\begin{tabular}{|c|c|c|}
\hline ct-compound & $\begin{array}{c}\text { concentration } \\
(\mu \mathrm{M})\end{array}$ & timepoint $(\mathrm{h})$ \\
\hline \multirow{3}{*}{$\mathrm{ct}-\mathrm{W}$} & 1.7 & 2 \\
\cline { 2 - 3 } & 0.6 & 4 \\
\cline { 2 - 3 } & 0.2 & 8 \\
\hline \multirow{2}{*}{-6 } & 0.6 & 24 \\
\cline { 2 - 3 } & 0.2 & 48 \\
\hline \multirow{2}{*}{ C-11 } & 1.7 & 8 \\
\cline { 2 - 3 } & 1.7 & 48 \\
\hline D-6 & 0.2 & 24 \\
\hline \multirow{2}{*}{ E-2 } & 1.7 & 8 \\
\cline { 2 - 3 } & 0.6 & 24 \\
\cline { 2 - 3 } & 0.2 & 48 \\
\hline
\end{tabular}

SI Table 11. CP50 values from CAPA experiments in HeLa and SH-SY5Y cell lines. AAV-H2GH HeLa cells or AAV-H2GH SH-SY5Y cells were incubated with the indicated compounds for $24 \mathrm{~h}$ in media containing $10 \%$ serum. Error represents the standard error from three independent replicates. Values correspond to data in main text Figure 6 and SI Figure 24.

\begin{tabular}{|c|c|c|}
\hline $\begin{array}{c}\text { ct- } \\
\text { compound }\end{array}$ & $\begin{array}{c}C P_{50}(\mu M) \\
A A V-H 2 G H \\
H e L a\end{array}$ & $\begin{array}{c}C P_{50}(\mu M) \\
A A V-H 2 G H \\
S H-S Y 5 Y\end{array}$ \\
\hline ct-W & $0.03 \pm 0.002$ & $0.26 \pm 0.19$ \\
\hline$E-1$ & $0.05 \pm 0.004$ & $0.06 \pm 0.003$ \\
\hline E-2 & $0.06 \pm 0.01$ & $0.14 \pm 0.07$ \\
\hline E-5 & $6.7 \pm 4.8$ & $0.19 \pm 0.06$ \\
\hline E-6 & $16.9 \pm 15.2$ & $0.30 \pm 0.09$ \\
\hline $\mathrm{C}-1$ & $0.07 \pm 0.01$ & --- \\
\hline C-12 & $0.02 \pm 0.004$ & --- \\
\hline C-13 & $8.4 \pm 6.8$ & --- \\
\hline C-14 & $0.94 \pm 0.17$ & --- \\
\hline
\end{tabular}


SI Table 12. $\mathrm{CP}_{50}$ values for ASOs with different $\mathbf{5}^{\prime}$ linkers in serum-free media. HGM HeLa cells were incubated with the indicated compounds for $24 \mathrm{~h}$ in serum-free media. Error represents the standard error from three independent replicates. Values correspond to data in SI Figure 19.

\begin{tabular}{|c|c|}
\hline ct-compound & $C_{50}(\mu \mathrm{M})$ \\
\hline ct-W & $0.002 \pm 0.0001$ \\
\hline A-6 & $0.50 \pm 0.11$ \\
\hline A-8 & $2.64 \pm 0.91$ \\
\hline B-5 & $0.13 \pm 0.08$ \\
\hline B-6 & $>20$ \\
\hline C-1 & $0.09 \pm 0.003$ \\
\hline C-13 & $>20$ \\
\hline C-12 & $0.02 \pm 0.002$ \\
\hline C-14 & $>20$ \\
\hline D-1 & $0.19 \pm 0.07$ \\
\hline D-3 & $>20$ \\
\hline D-2 & $0.38 \pm 0.27$ \\
\hline D-4 & $>20$ \\
\hline
\end{tabular}

SI Table 13. $\mathrm{CP}_{50}$ values for ASOs with different $\mathbf{5}^{\prime}$ linkers in full media. HGM HeLa cells were incubated with the indicated compounds for $24 \mathrm{~h}$ in media containing $10 \%$ serum. Error represents the standard error from three independent replicates. Values correspond to data in SI Figure 19.

\begin{tabular}{|c|c|}
\hline ct-compound & $C_{50}(\mu \mathrm{M})$ \\
\hline ct-W & $0.02 \pm 0.01$ \\
\hline A-6 & $0.08 \pm 0.02$ \\
\hline A-8 & $0.35 \pm 0.03$ \\
\hline B-5 & $0.08 \pm 0.02$ \\
\hline B-6 & $7.62 \pm 6.1$ \\
\hline C-1 & $0.07 \pm 0.01$ \\
\hline C-13 & $>20$ \\
\hline C-12 & $0.06 \pm 0.01$ \\
\hline C-14 & $7.2 \pm 3.5$ \\
\hline E-1 & $0.08 \pm 0.01$ \\
\hline E-5 & $10.5 \pm 8.0$ \\
\hline E-2 & $0.12 \pm 0.06$ \\
\hline E-6 & $3.7 \pm 1.6$ \\
\hline
\end{tabular}

SI Table 14. $\mathrm{CP}_{50}$ values for ASOs with $5^{\prime}$ amide and click linkers in serum-free media. HGM HeLa cells were incubated with the indicated compounds for $4 \mathrm{~h}$ in serum-free media. Error represents the standard error from three independent replicates. Values correspond to data in SI Figure 25.

\begin{tabular}{|c|c|}
\hline ct-compound & $\mathrm{CP}_{50}(\mu \mathrm{M})$ \\
\hline ct-W & $0.05 \pm 0.01$ \\
\hline C-11 & $1.37 \pm 0.13$ \\
\hline C-15 & $1.27 \pm 0.33$ \\
\hline B-4 & $1.51 \pm 0.23$ \\
\hline B-7 & $0.49 \pm 0.18$ \\
\hline A-6 & $3.98 \pm 0.21$ \\
\hline A-7 & $0.76 \pm 0.31$ \\
\hline
\end{tabular}

